\title{
LHC benchmarks from flavored gauge mediation
}

\section{N. lerushalmi, S. Iwamoto, G. Lee, V. Nepomnyashy and Y. Shadmi}

Physics Department, Technion - Israel Institute of Technology, Haifa 32000, Israel

E-mail: nivieru@technion.ac.il, sho@physics.technion.ac.il, leeg@physics.technion.ac.il, vera.nepomnyashy@gmail.com, yshadmi@physics.technion.ac.il

ABSTRACT: We present benchmark points for LHC searches from flavored gauge mediation models, in which messenger-matter couplings give flavor-dependent squark masses. Our examples include spectra in which a single squark - stop, scharm, or sup - is much lighter than all other colored superpartners, motivating improved quark flavor tagging at the LHC. Many examples feature flavor mixing; in particular, large stop-scharm mixing is possible. The correct Higgs mass is obtained in some examples by virtue of the large stop A-term. We also revisit the general flavor and CP structure of the models. Even though the A-terms can be substantial, their contributions to EDM's are very suppressed, because of the particular dependence of the A-terms on the messenger coupling. This holds regardless of the messenger-coupling texture. More generally, the special structure of the soft terms often leads to stronger suppression of flavor- and CP-violating processes, compared to naive estimates.

KeYwords: Supersymmetry Phenomenology

ARXIV EPRINT: 1603.02637 


\section{Contents}

1 Introduction 1

2 General structure: flavor and CP 4

3 MFV-like models: split top squark with and without charm mixing $\quad 8$

4 Non-MFV models $\quad 12$

$\begin{array}{lll}4.1 & \text { Large }\left(y_{u}\right)_{11} \text { : light up squark } & 13\end{array}$

$\begin{array}{lll}4.2 & \text { Large }\left(y_{u}\right)_{22} \text { : light charm squark } & 14\end{array}$

$\begin{array}{lll}4.3 & \text { Large }\left(y_{u}\right)_{32}: \text { light top and/or charm squark } & 16\end{array}$

$\begin{array}{lll}5 & \text { Conclusions } & 21\end{array}$

$\begin{array}{ll}\text { A FGM superpotential and symmetries } & 22\end{array}$

$\begin{array}{ll}\text { B Soft terms } & 23\end{array}$

$\begin{array}{ll}\text { C Higgs mass validation } & 25\end{array}$

$\begin{array}{ll}\text { D Calculation of flavor-violating parameters } & 25\end{array}$

\section{Introduction}

Flavored gauge mediation (FGM) models [1] extend minimal gauge mediation $[2,3]$ by introducing superpotential couplings between the messenger and matter superfields [4-8]. Generically, such couplings lead to flavor-dependent sfermion spectra. They also generate A-terms at the messenger-scale, allowing for a $125 \mathrm{GeV}$ Higgs with relatively light squarks [9-21]. The main observation of [1] is that any concrete mechanism for generating the standard model (SM) Yukawas also governs the textures of the messenger-matter couplings, and can therefore give spectra consistent with low-energy bounds. The messenger coupling is either similar in structure to the corresponding SM Yukawa texture, resulting in minimal flavor violation (MFV)-like models in which only the third-generation sfermion masses are affected (with the possiblility of large mixings between different generations of sfermions), or it has an altogether different structure, leading to large effects in the firstand second-generation sfermions. Flavor constraints on MFV-like models were analyzed in detail in [22, 23]. Non-MFV models were explored in [24], and shown to give unusual squark spectra, featuring for example a single up or charm squark much lighter than the remaining squarks, and/or large stop-scharm mixing, with important implications for LHC supersymmetry searches [25-27]. Flavor effects in different extensions of gauge mediated supersymmetry breaking (GMSB) models were also considered in [28-31]. 
Our main goal in this paper is to extend the qualitative analysis of [24], and to provide interesting FGM benchmarks for the LHC run II. We derive several representative examples by starting with specific flavor models at the high scale, and including RGE effects above and below the messenger scale. We find that even MFV-like models have a rich structure which allows for large stop hierarchies and a large stop mixing. Examples of this type were also discussed recently in [32]. Our examples typically feature the gluino and most squarks at or above $2 \mathrm{TeV}$, with one or two squarks at or below $1 \mathrm{TeV}$. Flavor mixing generically occurs in parts of the spectra. Many of these examples, including all the MFV-like models and some of the non-MFV models, also give the correct Higgs mass.

Regardless of the texture of the messenger-matter coupling, the fact that the soft terms are all determined by a single new coupling matrix (in addition to the gauge and SM Yukawa couplings) leads to several remarkable features in the flavor and CP structure of the models. First, the A-terms do not generate electric dipole moments (EDMs) at leading order because they only depend on the absolute value squared of the messenger coupling [23]. Thus, the lack of a supersymmetric CP problem, one of the nicest features of GMSB, is not spoiled in these models. This holds more generally for other extensions of GMSB that introduce a single dominant messenger-SM-SM coupling. Second, this special structure also results in extra protection against flavor-non-diagonal $\mathrm{CP}$ violation. In particular, if there is a single non-zero entry in the messenger coupling matrix, it does not introduce any new $\mathrm{CP}$-violating phase, and the only $\mathrm{CP}$ violation originates from the $\mathrm{SM}$ Yukawas. In the LL sector, the only source of CP violation is therefore the CKM matrix, and $\mathrm{CP}$ - and flavor-violating processes in the first and second generations are very small. Finally, in models with a vanishing (or small) down-type messenger coupling, the new contributions to the R-down squark masses involve two powers of the SM down Yukawa, so these masses are barely modified from their GMSB values. The constraints on the combination of LL and RR flavor parameters in the down sector, which are particularly stringent, are therefore automatically met.

An important ingredient in FGM models is a mechanism for generating the SM Yukawas. As we review in section 2, since the SM Yukawas have a hierarchical structure, the messenger-matter couplings are generically hierarchical too, with at most a single entry being $\mathcal{O}(1)$. The existence and location of this entry in the messenger-matter coupling matrix depends on the details of the model. As a concrete example, we assume that fermion masses are governed by a horizontal U(1) symmetry, broken by a spurion $\lambda \sim 0.2$ of charge -1 . The fermion masses are then proportional to different powers of the spurion $\lambda$, with the powers determined by the flavor charges of the matter and Higgs fields. Once the messenger flavor charge is specified, the messenger-matter coupling is completely determined too. There are then three possibilities:

1. The messenger charge is the same as the Higgs charge. Then the texture of the messenger-coupling matrix $y_{U}$ is identical to the Yukawa texture $Y_{U}$, and only its $3-3$ entry is order one. This leads to the MFV-like models of $[1,16]$. Since the only large effects are in the third generation, the models are consistent with low-energy constraints. 
2. The messenger charge is larger than the Higgs charge. Then the messenger couplings have larger suppressions compared to the Yukawa couplings, $y_{U i j}<Y_{U i j}$. Since the messenger couplings enter squared in the soft terms, their effects on the masses are very small, and again, the models are consistent with flavor bounds. Note that, even though the mass splittings are small in this case, flavor mixing can be large. The reason is that the dominant contribution to sfermion masses is the gauge-mediated contribution, which is proportional to the identity. The mixing is therefore solely determined by the corrections to the GMSB contribution $[1,33]$.

3. The messenger charge is smaller than the Higgs charge. Then one of the messenger couplings involving the first or second generation can be $\mathcal{O}(1)$, giving a large mass splitting to the squarks of the respective generation. This requires precise down alignment in order to satisfy flavor constraints.

In the examples below, we choose different messenger charges, thus sampling different entries of the messenger coupling matrix, and covering most of the FGM parameter space. In each case, one can get a viable spectrum consistent with flavor constraints.

Note that, by construction, all the models exhibit some degree of supersymmetric alignment [16]: the sfermion mass matrices and the SM Yukawa matrices are simultaneously diagonal up to corrections of order $\lambda \sim 0.2$. This alignment is sufficient to satisfy flavor constraints in models of types (1) and (2). The non-MFV models further require zero 1-2 L-down mixing. We refer to this alignment as supersymmetric alignment $[16,24]$ because the supersymmetric messenger couplings, rather than the supersymmetry-breaking parameters [34], are controlled by the flavor symmetry. As a result, the flavor symmetry is manifest at the (possibly) low messenger scale. A generic feature of these models is $O(\lambda)$ mixing in the first and second generation L-squarks, either in the up sector only [35], or, in models of type (1) and (2), in both the up and down sector.

We present several examples with non-trivial squark spectra Our benchmark points (BPs) have negligible messenger-lepton couplings, so the slepton masses remain degenerate as in GMSB. The NLSP in these examples is either a bino or an R-stau. The latter possibility can be realized even in models with a single messenger pair, since the splitting in the squark masses due to the new messenger coupling feeds into the slepton masses through the hypercharge RGE contribution [9, 16]. As usual in GMSB models, the NLSP lifetime depends on the gravitino mass, which in turn depends on the underlying supersymmetrybreaking scale. In the examples we show, this uncertainty translates into a wide range of bino or R-slepton lifetimes, underscoring the importance of searches for intermediate non-prompt decays (see also [36] for a recent discussion).

Since our main focus is squark flavor, we also show examples in which the Higgs has a mass below $125 \mathrm{GeV}$, requiring an additional modification of the model to raise it, such as the addition of an NMSSM singlet. Such modifications will have little effect on the sfermion flavor structure. The large Higgs mass in our models is driven by the large stop LR mixing, and in some examples, by the large stop masses. To get a handle on the uncertainty in the calculated Higgs mass, we computed it using both SOFTSUSY [37] and FeynHiggs [3842]. We collect the comparisons between the two codes in appendix C. Furthermore, the 
large stop-scharm mixing and the large 3-2 entry of the up A-term featured in some of the models may modify the Higgs mass by a few GeV [43-46]. Thus, the large Higgs mass values we obtain in the models should mainly be viewed as an indication that the models can accommodate a correct Higgs mass.

This paper is organized as follows. In section 2 we discuss the general flavor and CP structure of the models. In section 3 we present several examples of MFV-like models, and the resulting low energy spectra. Non-MFV examples are presented in section 4 . We conclude with some remarks in section 5. Appendix A reviews some basics of FGM models, including possible symmetries yielding the FGM superpotential, and the issue of superpotential and Kähler mixing. Our conventions for the soft terms, as well as the full expressions for the soft terms in the presence of up-type messenger couplings, are collected in appendix B. In appendix $\mathrm{C}$ we compare the values of the Higgs mass as computed by SOFTSUSY and FeynHiggs. The values of the flavor-violating $\delta$ 's for our different examples are collected in appendix D.

\section{General structure: flavor and CP}

We consider models with the superpotential $[1,5]$

$$
\begin{aligned}
W=\sum_{I=1}^{N_{5}} X\left(T_{I} \bar{T}_{I}+D_{I} \bar{D}_{I}\right)+\bar{D}_{1} q y_{U} u^{c}+D_{2} q y_{D} d^{c}+D_{2} l y_{L} e^{c} \\
+H_{U} q Y_{U} u^{c}+H_{D} q Y_{D} d^{c}+H_{D} l Y_{L} e^{c} .
\end{aligned}
$$

Here $X$ parameterizes the supersymmetry breaking with $\langle X\rangle=M+F \theta^{2},\left(T_{I}, \bar{T}_{I}\right)$ and $\left(D_{I}, \bar{D}_{I}\right)$ are respectively $\mathrm{SU}(3)$ triplet and $\mathrm{SU}(2)$ doublet messenger pairs, and $N_{5}$ is the number of messenger pairs. $Y_{U, D, L}$ are the SM Yukawa coupling matrices, and $y_{U, D, L}$ are the analogous $3 \times 3$ flavor-space matrices of messenger-matter couplings; hereafter we use instead the complex conjugates of these couplings,

$$
Y_{u, d, l}=\left(Y_{U, D, L}\right)^{*}, \quad y_{u, d, l}=\left(y_{U, D, L}\right)^{*},
$$

for easier comparison with the SM flavor constraints. Our main focus below is on examples with $y_{d}=y_{l}=0$, but in this section we discuss some general features of the models with both $y_{u}$ and $y_{d}$ present. The form of the superpotential (2.1) can be enforced by various choices of global symmetries, as we review in appendix A. The expressions for the soft terms, for $y_{d}=y_{l}=0$, are collected in appendix B. For simplicity, we denote $\bar{D} \equiv \bar{D}_{1}$.

The new couplings generate one-loop A-terms at the messenger scale as well as new contributions to the sfermion soft masses. Unlike the GMSB contributions, these are generically flavor dependent, and contain new sources of $\mathrm{CP}$ violation. In table 1 , we summarize the mass insertion approximation (MIA) estimates for the most stringently constrainted squark flavor parameters from [24, 47]. Working in terms of the physical masses and mixings, we define [48]

$$
\left(\delta_{i j}^{q}\right)_{\mathrm{MM}}=\frac{\Delta \tilde{m}_{j i}^{2}}{\tilde{m}_{q}^{2}}\left(K_{M}^{q}\right)_{i j}\left(K_{M}^{q}\right)_{j j}^{*},
$$




\begin{tabular}{|cc|ccc|}
\hline$q$ & $i j$ & $\left|\left(\delta_{i j}^{q}\right)_{\mathrm{MM}}\right|$ & $\sqrt{\operatorname{Im}\left[\left(\delta_{i j}^{q}\right)_{\mathrm{MM}}^{2}\right]}$ & $\sqrt{\operatorname{Im}\left[\left(\delta_{i j}^{q}\right)_{L L}\left(\delta_{i j}^{q}\right)_{R R}\right]}$ \\
\hline$d$ & 12 & 0.07 & 0.01 & 0.0005 \\
$u$ & 12 & 0.1 & 0.05 & 0.003 \\
$d$ & 23 & 0.6 & 0.2 & 0.07 \\
\hline
\end{tabular}

Table 1. Bounds on $\left(\delta_{i j}^{q}\right)_{\mathrm{MM}}$ for $1 \mathrm{TeV}$ common squark and gluino masses. For higher squark masses the bounds scale approximately as $\tilde{m}_{q}$, with a weaker dependence on the gluino mass.

where $\Delta \tilde{m}_{j i}^{2}=m_{\tilde{q}_{j}}^{2}-m_{\tilde{q}_{i}}^{2}$ is the squared-mass difference of the relevant squarks, $\tilde{m}_{q}^{2}=$ $\frac{1}{3} \sum_{\alpha=1}^{3} m_{\tilde{q}_{\alpha}}^{2}$ is the average squark squared-mass, and $\left(K_{M}^{q}\right)_{i j}$ is the mixing appearing in the quark-squark-gluino coupling. In the models considered below, there is a single dominant source of flavor violation, so the MIA gives a reasonable estimate. As is well known, this approximation breaks down with $\mathcal{O}(1)$ mass differences (see for example [35, 49] for recent analyses). In some of the examples below, the relative mass splitting is very small, so the MIA is reliable. In the others, with $\mathcal{O}(1)$ mass differences, the mixings are very small, so that even though the MIA does not give a good estimate, flavor constraints are satisfied thanks to the small mixings.

The flavor textures of the soft terms are not arbitrary in these models. Rather, they are given by specific combinations of the messenger couplings and the SM Yukawas, which can be determined by a spurion analysis, treating the matrices $Y_{u}, y_{u}$, etc. as spurions of the $\mathrm{SU}(3)^{5}$ flavor symmetry [1]. Furthermore, any power of the messenger coupling appearing in the soft terms, say $y_{u}$, must be accompanied by the same power of $y_{u}^{\dagger}$ [23]. To see this, one can invoke a global U(1) under which only the messenger field $\bar{D}_{1}$ is charged. $y_{u}$ is the only spurion breaking this $\mathrm{U}(1)$. Since the soft terms are singlets of this symmetry, $y_{u}$ must appear with $y_{u}^{\dagger}$. This special structure ameliorates some of the constraints on the models, especially in the presence of a single new coupling, as we now discuss.

First, the models do not suffer from the flavor-diagonal supersymmetric CP problem. Indeed, one of the virtues of GMSB models is that they do not introduce large quark and lepton EDMs. In minimal GMSB, the A-terms are only generated from the gaugino mass through the RGE, so there is no relative phase between them. Naively, this seems to be spoiled in FGM models (and more generally in models with messenger-matter or messengerHiggs couplings), since the messenger-matter couplings generate A-terms at the messenger scale. However, to leading order, these A-terms are real [23]. For example, consider the $y_{u}$ contribution to $A_{d}$ at one-loop order. It is determined by the spurion analysis described above as

$$
A_{d}^{*} \propto y_{u} y_{u}^{\dagger} Y_{d}
$$

To discuss the resulting EDMs, we rotate the squark superfields into the quark mass basis, in which the SM Yukawa matrices are diagonal. In this basis, the A-term is given by

$$
V_{d L} A_{d}^{*} V_{d R}^{\dagger} \propto V_{d L} y_{u} y_{u}^{\dagger} V_{d L}^{\dagger} Y_{d}^{\operatorname{diag}}
$$


where $V_{d L}, V_{d R}\left(V_{u L}, V_{u R}\right)$ are the matrices that bidiagonalize the down and up SM Yukawa matrices,

$$
Y_{u}^{\text {diag }}=V_{u L} Y_{u} V_{u R}^{\dagger}, \quad Y_{d}^{\text {diag }}=V_{d L} Y_{d} V_{d R}^{\dagger},
$$

such that the CKM matrix is given by $V_{\mathrm{CKM}}=V_{u L} V_{d L}^{\dagger}$. Since $V_{d L} y_{u} y_{u}^{\dagger} V_{d L}^{\dagger}$ is hermitian,

$$
\left(V_{d L} A_{d}^{*} V_{u R}^{\dagger}\right)_{11} \propto\left(V_{d L} y_{u} y_{u}^{\dagger} V_{d L}^{\dagger}\right)_{11}\left(Y_{d}^{\text {diag }}\right)_{11}
$$

has no complex phase and does not generate an EDM. Similarly, the $y_{u}$ contribution to $A_{u}$ is restricted to be of the form

$$
\left(y_{u} y_{u}^{\dagger}\right) Y_{u}, \quad Y_{u}\left(y_{u}^{\dagger} y_{u}\right)
$$

and thus $\left(A_{u}\right)_{11}$ evaluated in the fermion mass basis is real. We can repeat this discussion for the contributions from $y_{d}$. The same reasoning also applies to any model with a messenger-SM-SM coupling. A non-zero EDM can, in principle, be generated at higher orders in the messenger loops, coming either from higher-loop corrections to the A-terms, or from multiple insertions of $\delta_{L R}, \delta_{L L}, \delta_{R R}$, but these are very small. ${ }^{1}$

We now turn to flavor violation. As noted above, the combination of flavor violation in the L- and R-down squarks is tightly constrained by experiment. These constraints are alleviated in our models for $y_{d}=0$, which can be achieved by a choice of global symmetries (see appendix A). With no down-type messenger couplings, the new contribution to the RR down squark mass matrix eq. (B.16), is suppressed by two powers of the down Yukawa. In the fermion mass basis, this mass matrix is given by

$$
V_{d R}\left(\delta \tilde{m}_{d_{R}}^{2}\right) V_{d R}^{\dagger}
$$

Thus for example, its 1-2 entry is

$$
\left(V_{d R}\left(\delta \tilde{m}_{d_{R}}^{2}\right) V_{d R}^{\dagger}\right)_{12} \sim \frac{m_{d} m_{s}}{\left\langle H_{D}\right\rangle^{2}}\left(V_{d L} y_{u} y_{u}^{\dagger} V_{d L}^{\dagger}\right)_{12} \sim 10^{-8} \tan ^{2} \beta\left(V_{d L} y_{u} y_{u}^{\dagger} V_{d L}^{\dagger}\right)_{12},
$$

where $m_{d}\left(m_{s}\right)$ is the down (strange) quark mass and $\left\langle H_{D}\right\rangle$ is the vacuum expectation value of $H_{D}$. This is negligible even for an arbitrary $\mathcal{O}(1)$ matrix $y_{u}$, so that the $\mathrm{R}$ down squarks are nearly degenerate.

The remaining LL and RR mass matrices involve various combinations of $y_{u}$ and the SM Yukawas. First, consider terms that only involve $y_{u}$. In the fermion mass basis, these have the form

$$
\begin{aligned}
& \tilde{m}_{d_{L}}^{2} \supset\left(V_{d L} W_{u L}^{\dagger}\right)\left[\left(y_{u}^{\mathrm{diag}} y_{u}^{\mathrm{diag}^{\dagger}}\right)+\#\left(y_{u}^{\mathrm{diag}} y_{u}^{\mathrm{diag}^{\dagger}}\right)^{2}\right]\left(V_{d L} W_{u L^{\dagger}}\right)^{\dagger}, \\
& \tilde{m}_{U_{L}}^{2} \supset\left(V_{u L} W_{u L^{\dagger}}\right)\left[\left(y_{u}^{\mathrm{diag}} y_{u}^{\mathrm{diag}^{\dagger}}\right)+\#\left(y_{u}^{\mathrm{diag}} y_{u}^{\mathrm{diag}^{\dagger}}\right)^{2}\right]\left(V_{u L} W_{u L^{\dagger}}\right)^{\dagger} \\
& \tilde{m}_{U_{R}}^{2} \supset\left(V_{u R} W_{u R^{\dagger}}^{\dagger}\right)\left[\left(y_{u}^{\text {diag }^{\dagger}} y_{u}^{\text {diag }}\right)+\#\left(y_{u}^{\text {diag }}{ }^{\dagger} y_{u}^{\text {diag }}\right)^{2}\right]\left(V_{u R} W_{u R}\right)^{\dagger} \text {, }
\end{aligned}
$$

\footnotetext{
${ }^{1}$ Many of the possible structures are actually real. In fact, with $y_{d}=0$, no imaginary part can be generated if $y_{u}$ is rank 1 .
} 
where the \# are real numbers, and we define

$$
y_{u}^{\text {diag }} \equiv W_{u L} y_{u} W_{u R}^{\dagger}
$$

with $y_{u}^{\text {diag }}$ diagonal. Clearly, flavor mixings are determined by the misalignment between $y_{u}$ and the relevant Yukawa matrix. The most stringent constraint is on $\left(\delta_{12}^{d}\right)_{L L}$. In the following, we will consider two types of models.

- In MFV-like models, $\left(y_{u}\right)_{i j}$ and $\left(Y_{u}\right)_{i j}$ are the same up to order-one numbers, for any $i, j$. Then the only large entry of $y_{u}$ is the 33 entry, with

$$
y_{u}^{\operatorname{diag}} y_{u}^{\operatorname{diag}^{\dagger}} \sim \operatorname{diag}\left(0, \lambda^{4}, 1\right)
$$

where $\lambda \sim 0.2$. Also, without accidental cancellations or enhancements, $\left(W_{u L} V_{d L}^{\dagger}\right)_{i j}$ is expected to be the same order as $\left(V_{\mathrm{CKM}}\right)_{i j}$. Then $\left(\delta_{12}^{d}\right)_{L L} \sim \lambda^{5}$, which is at the level of experimental constraints, and $\left(\delta_{23}^{d}\right)_{L L} \sim \lambda^{2}$, which is below current sensitivity. The MFV-like models are thus consistent with flavor bounds on the down sector.

As for the up sector, in MFV-like models the 1-2 up block is approximately degenerate, and this degeneracy is sufficient for satisfying all flavor constraints. Furthermore, generically in these models $V_{u L} \sim W_{u L}$ and $W_{u R} \sim V_{u R}$, so that

$$
\left(V_{u L} W_{u L}^{\dagger}\right) \sim V_{u L}, \quad\left(V_{u R} W_{u R}^{\dagger}\right) \sim V_{u R} .
$$

- In other models, $y_{u}$ has $\mathcal{O}(1)$ entries in the first or second row or column. Then $y_{u}^{\text {diag }} y_{u}^{\text {diag }}{ }^{\dagger}$ has $\mathcal{O}(1)$ entries in its 1-2 block, and precise alignment of the down squark and quark matrices is required for $\left(V_{d L} W_{u L}^{\dagger}\right)_{12}$ to be small. We therefore construct models in which the down Yukawa is approximately diagonal, and $y_{u} y_{u}^{\dagger}$ is approximately diagonal as well. Then, $V_{d L}, W_{u L}$, and $W_{u R}$ are close to the identity matrix, and flavor mixing arises predominantly from the SM mixing matrices $V_{u L} \sim V_{\mathrm{CKM}}$ and $V_{u R}$. New sources of CP-violating phases are therefore suppressed.

Both types of models will be realized below using a horizontal symmetry that also generates the SM fermion masses. As a result, in each case, only a single entry of $y_{u}$ is $\mathcal{O}(1)$. This leads to a further suppression of the imaginary parts of $\left(\delta_{i j}\right)_{\mathrm{MM}}$, since the $\mathcal{O}(1)$ entry only enters in its absolute value squared.

As mentioned above, the soft mass matrices also contain certain combinations of $y_{u}$ and the SM Yukawas. It is easy to verify that these do not introduce any new qualitative features in the models considered below. In the MFV-like models of section $3, y_{u} \sim Y_{u}$, so the discussion above still holds. In particular, the texture of $y_{u}$ is unaltered when rotating to the up mass basis. In the non-MFV models of sections 4.1 and 4.2, these mixed terms are subdominant because there is no large overlap between $y_{u}$ and $Y_{u}$. Such overlap is present in the models of section 4.3 , but as we will see, flavor constraints are met in these models roughly as discussed above. We stress that, for the numerical examples we present in section 3 and section 4, the full expressions of the soft terms are taken into account. 


\section{MFV-like models: split top squark with and without charm mixing}

As discussed above, models in which $\left(y_{u}\right)_{i j}$ and $\left(Y_{u}\right)_{i j}$ are the same up to $\mathcal{O}(1)$ numbers are consistent with flavor experiments. This possibility is realized in models in which the fermion masses are explained by a horizontal symmetry, if the messenger $\bar{D}$ has the same flavor charge as $H_{U}$. We thus consider a $\mathrm{U}(1)$ horizontal symmetry with a spurion $\lambda$ of charge -1 , and matter field charges

$$
Q_{1}(5), Q_{2}(4), Q_{3}(2), u_{1}(1), u_{2}(-1), u_{3}(-2), d_{1}(1), d_{2}(0), d_{3}(0)
$$

with the Higgses and $\bar{D}$ messenger neutral. The Yukawa matrices are then

$$
y_{u} \sim Y_{u} \sim\left(\begin{array}{ccc}
\lambda^{6} & \lambda^{4} & \lambda^{3} \\
\lambda^{5} & \lambda^{3} & \lambda^{2} \\
\lambda^{3} & \lambda & 1
\end{array}\right), \quad Y_{d} \sim\left(\begin{array}{ccc}
\lambda^{6} & \lambda^{5} & \lambda^{5} \\
\lambda^{5} & \lambda^{4} & \lambda^{4} \\
\lambda^{3} & \lambda^{2} & \lambda^{2}
\end{array}\right),
$$

where, as explained above, $\sim$ means that the matrices are given up to $\mathcal{O}(1)$ coefficients. These lead to the fermion mixing matrices,

$$
\begin{aligned}
V_{u L} \sim\left(\begin{array}{ccc}
1 & \lambda & \lambda^{3} \\
\lambda & 1 & \lambda^{2} \\
\lambda^{3} & \lambda^{2} & 1
\end{array}\right), & V_{u R} \sim\left(\begin{array}{ccc}
1 & \lambda^{2} & \lambda^{3} \\
\lambda^{2} & 1 & \lambda \\
\lambda^{3} & \lambda & 1
\end{array}\right), \\
V_{d L} \sim\left(\begin{array}{ccc}
1 & \lambda & \lambda^{3} \\
\lambda & 1 & \lambda^{2} \\
\lambda^{3} & \lambda^{2} & 1
\end{array}\right), & V_{d R} \sim\left(\begin{array}{ccc}
1 & \lambda & \lambda \\
\lambda & 1 & 1 \\
\lambda & 1 & 1
\end{array}\right),
\end{aligned}
$$

with $V_{d L} \sim V_{u L} \sim V_{\mathrm{CKM}}$,

$$
V_{\mathrm{CKM}}=V_{u L} V_{d L}^{\dagger} \sim\left(\begin{array}{ccc}
1 & \lambda & \lambda^{3} \\
\lambda & 1 & \lambda^{2} \\
\lambda^{3} & \lambda^{2} & 1
\end{array}\right)
$$

The corrections to the squark soft mass terms have the structure

$$
\delta \tilde{m}_{q}^{2} \sim\left(\begin{array}{ccc}
\lambda^{6} & \lambda^{5} & \lambda^{3} \\
\lambda^{5} & \lambda^{4} & \lambda^{2} \\
\lambda^{3} & \lambda^{2} & 1
\end{array}\right), \quad \delta \tilde{m}_{u R}^{2} \sim\left(\begin{array}{ccc}
\lambda^{6} & \lambda^{4} & \lambda^{3} \\
\lambda^{4} & \lambda^{2} & \lambda \\
\lambda^{3} & \lambda & 1
\end{array}\right),
$$

while

$$
A_{u}^{*} \sim\left(\begin{array}{ccc}
\lambda^{6} & \lambda^{4} & \lambda^{3} \\
\lambda^{5} & \lambda^{3} & \lambda^{2} \\
\lambda^{3} & \lambda & 1
\end{array}\right)
$$

so a large stop A-term is possible.

Although the above expressions are modified by RGE effects, they provide first estimates of the flavor-violating terms in the models. As discussed in the previous section, the down-RR entries are negligible in these models. The remaining entries are shown in table 2. 


\begin{tabular}{|cc|ccc|}
\hline$q$ & $i j$ & $\left|\left(\delta_{i j}^{q}\right)_{L L}\right|$ & $\left|\left(\delta_{i j}^{q}\right)_{R R}\right|$ & $\sqrt{\left|\left(\delta_{i j}^{q}\right)_{L L}\right|\left|\left(\delta_{i j}^{q}\right)_{R R}\right|}$ \\
\hline $\mathrm{d}$ & 12 & $\lambda^{5}$ & - & - \\
$\mathrm{u}$ & 12 & $\lambda^{5}$ & $\lambda^{4}$ & $\lambda^{9 / 2}$ \\
$\mathrm{~d}$ & 23 & $\lambda^{2}$ & - & - \\
\hline
\end{tabular}

Table 2. Parametric estimates of $\left(\delta_{i j}^{q}\right)_{\mathrm{MM}}$ in MFV-like models. Omitted entries are negligible.

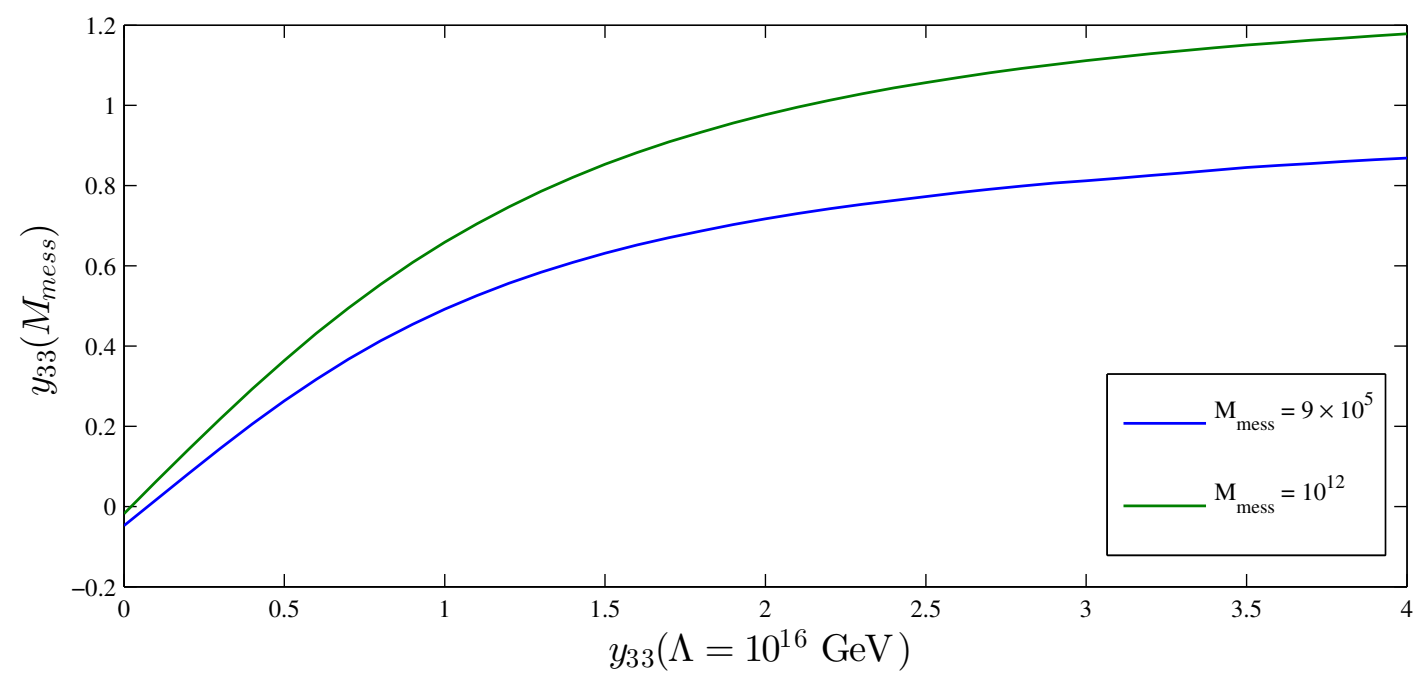

Figure 1. $\left(y_{u}\right)_{33}$ at the messenger scale $M$ as a function of its value at the flavor scale $\Lambda$, for $\left(y_{u}\right)_{32}(\Lambda)=0.2$.

With no CP violating phases, all of these are significantly below the experimental bounds. In the presence of CP violation, the product $\left(\delta_{i j}^{q}\right)_{L L}\left(\delta_{i j}^{q}\right)_{R R}$ is of order the bound quoted in table 1. However, in the following examples, this product is also below the bound even for $\mathcal{O}(1)$ phases, mainly because the typical gluino and average squark masses are higher than $1 \mathrm{TeV}$.

In both $\delta \tilde{m}_{q}^{2}$ and $\delta \tilde{m}_{u R}^{2}$, the dominant contribution is the $3-3$ entry. The second important contribution is $\left(\delta \tilde{m}_{u R}^{2}\right)_{32}$. These contributions come from $\left(y_{u}\right)_{33}$ and $\left(y_{u}\right)_{32}$, so the squark spectra are particularly sensitive to the $\mathcal{O}(1)$ coefficients of these two entries. If the flavor scale, $\Lambda$, is much higher than the the messenger scale $M$, these couplings may be significantly modified by the running. In figure 1 , we show $\left(y_{u}\right)_{33}(M)$ as a function of $\left(y_{u}\right)_{33}(\Lambda)$ for a given value of $\left(y_{u}\right)_{32}(\Lambda)=0.2$ and $\Lambda=10^{16} \mathrm{GeV}$. The value of $\left(y_{u}\right)_{33}$ is clearly decreased by the running. In particular, large boundary values of $\left(y_{u}\right)_{33}(\Lambda)$, say above 2 , flow to $\left(y_{u}\right)_{33} \sim 1$ at the messenger scale. A milder decrease, with no IR fixed point behavior, is seen in the running of $\left(y_{u}\right)_{32}$, which we display in figure 2 .

In the following, we discuss two concrete examples of MFV-like models; one with a low messenger scale, $M=9.0 \times 10^{5} \mathrm{GeV}$, and the other with a high messenger scale, $M=10^{12} \mathrm{GeV}$. The models are obtained by choosing specific $\mathcal{O}(1)$ coefficients of the entries of $y_{u}$ at the flavor scale $\Lambda=10^{15} \mathrm{GeV}$. The various couplings are then evolved to the 


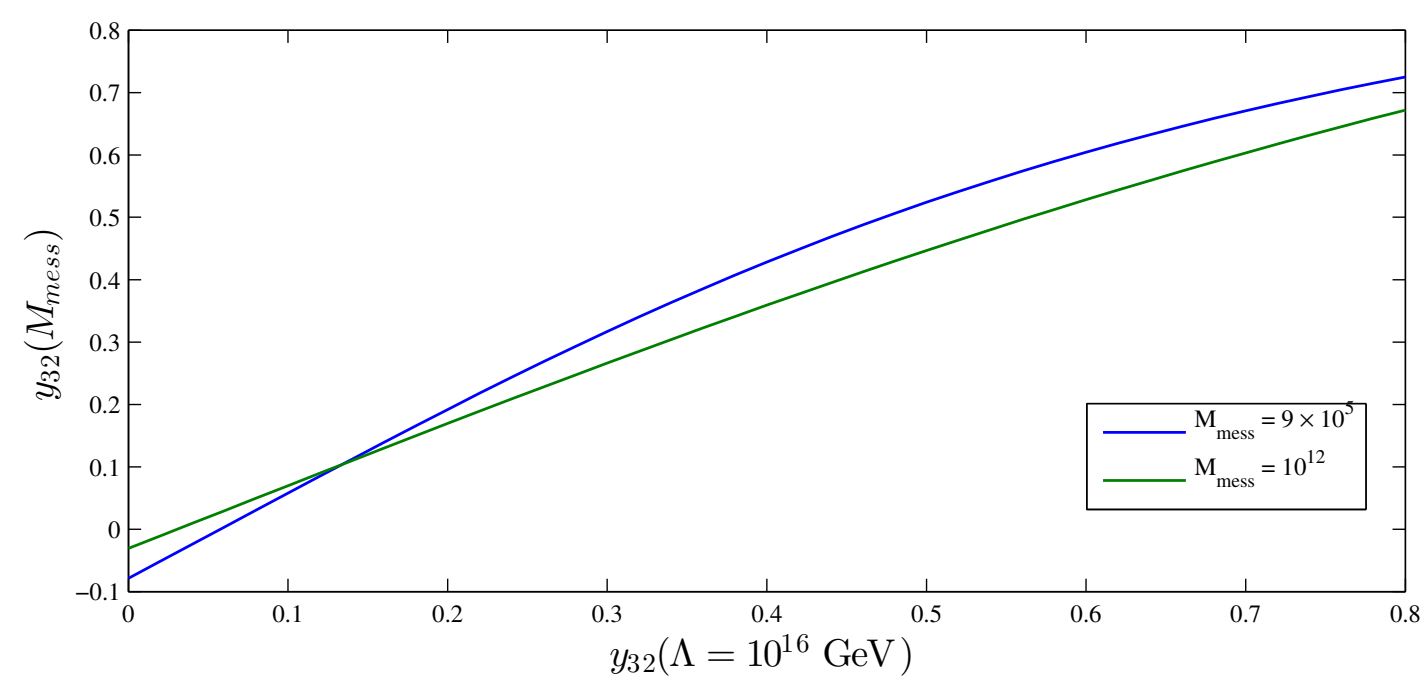

Figure 2. $\left(y_{u}\right)_{32}$ at the messenger scale as a function of $\left(y_{u}\right)_{32}$ at the $\Lambda$ scale, for $\left(y_{u}\right)_{33}(\Lambda)=1$.

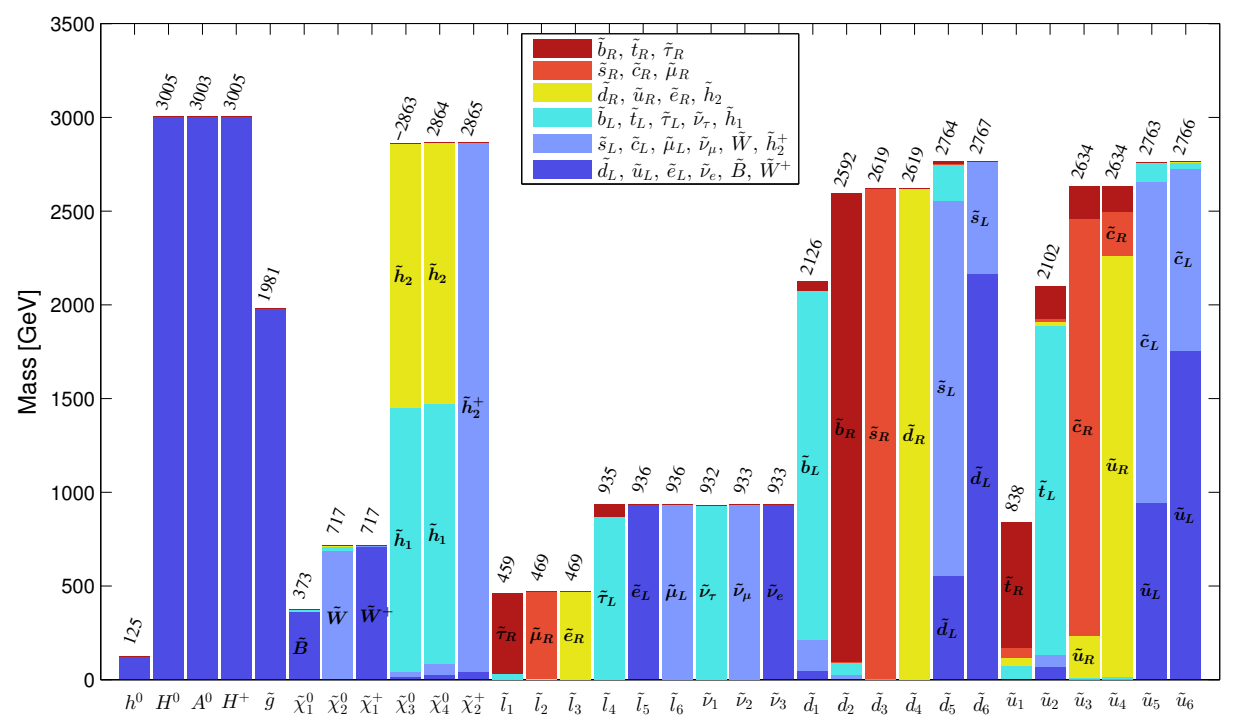

Figure 3. Spectrum for BP MFV-t with large $\left(y_{u}\right)_{33}\left(\Lambda=10^{15} \mathrm{GeV}\right)=2.9$ and scales $M=$ $9 \times 10^{5} \mathrm{GeV}, F / M=2.7 \times 10^{5} \mathrm{GeV}$. Mixings are shown for sfermion and gaugino mass eigenstates. In this model, we find $\mu=2870 \mathrm{GeV}$ and $\left(A_{u}\right)_{33}=-1920 \mathrm{GeV}$.

messenger scale $M$, where the soft masses are calculated and fed as input to SOFTSUSY [37], which is used to obtain the low-energy spectra.

The first BP "MFV-t", where "t" represents the stop-like lightest squark, is obtained with the scales $M=9.0 \times 10^{5} \mathrm{GeV}$ and $F / M=2.7 \times 10^{5} \mathrm{GeV}$, and is shown in figure 3 . At the messenger scale, $\left(y_{u}\right)_{33}(M)=0.8$. The Higgs mass is $125 \mathrm{GeV}$, the R-stop is at $\sim 840 \mathrm{GeV}$, and the L-stop and L-sbottom are near $2.1 \mathrm{TeV}$. The remaining squarks have masses between 2.5 to $2.8 \mathrm{TeV}$, and the gluino is at $2 \mathrm{TeV}$. The NLSP is the bino, which decays promptly with $c \tau_{\tilde{B}}>0.09 \mathrm{~mm}$ if the $F$ term of $X$ is the dominant $F$-term in the 


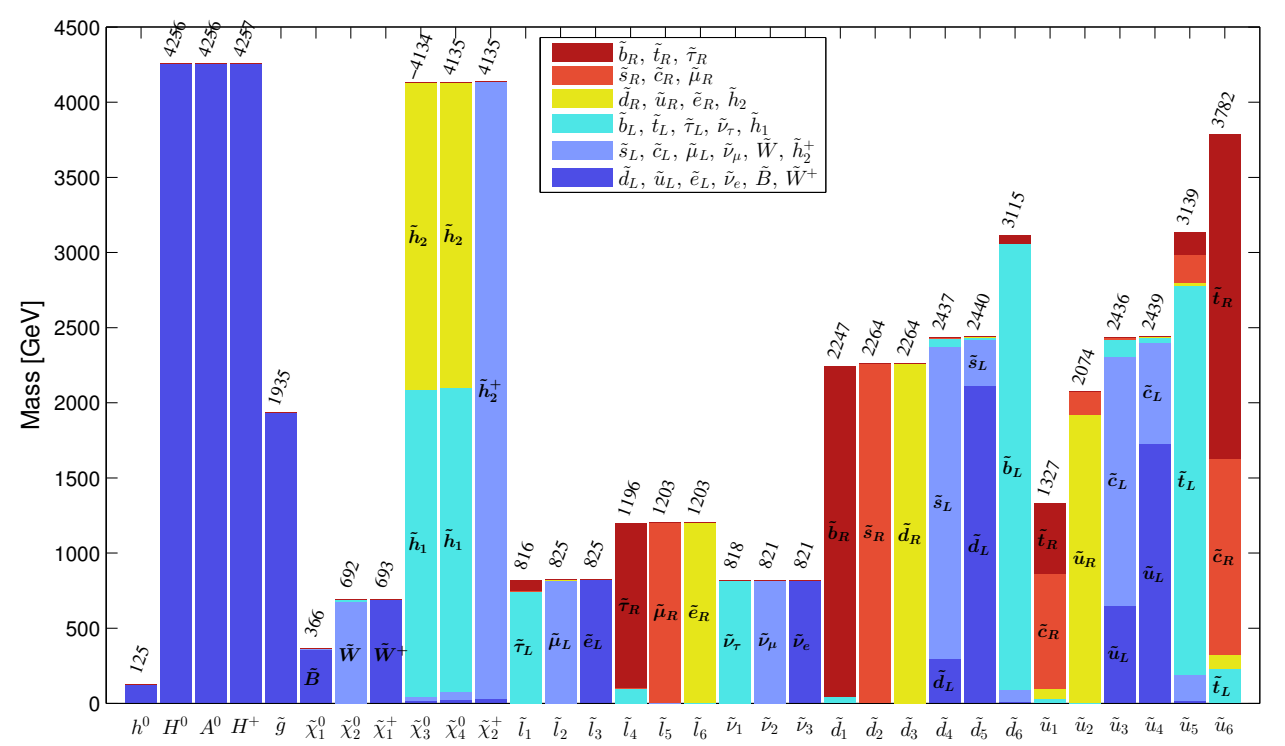

Figure 4. Spectrum for BP MFV32 with large $\left(y_{u}\right)_{32}\left(\Lambda=10^{15} \mathrm{GeV}\right)=0.63$ and scales $M=$ $10^{12} \mathrm{GeV}, F / M=2.71 \times 10^{5} \mathrm{GeV}$. Mixings are shown for sfermion and gaugino mass eigenstates. In this model, we find $\mu=4150 \mathrm{GeV}$ and $\left(A_{u}\right)_{33}=-2390 \mathrm{GeV}$.

theory. Thus, the correct Higgs mass is obtained with squarks that are still within the reach of the LHC.

From the point of view of mass hierarchies, this spectrum is not unusual, with the first- and second-generation squarks approximately degenerate; however, the L-squark mass eigenstates are mixtures of up-charm or down-strange states, with the size of the mixing of order of the Cabbibo mixing. Such mixings are completely generic whenever the squarks are not exactly degenerate because of the fermion Cabbibo mixing. Note that substantial 1-2 mixing can arise even when the mass splittings are small, since the leading contribution to the first- and second-generation squark masses is the flavor-diagonal GMSB contribution [33]. It would be interesting to explore whether this mixing leads to observable effects at the LHC as charm tagging is improved.

As noted above, the only potentially dangerous source of flavor violation in our examples involves the product of the imaginary parts of the LL and RR 1-2 entries in the up sector. We list these (for this example and the following examples) in appendix D. For the spectrum of figure 3 , the bounds of table 1 should be rescaled by $\sim 2$. The model is thus viable even with $\mathcal{O}(1)$ phases.

In this example, the stop-scharm mixing is quite small. As discussed above, this mixing is sensitive to the $\mathcal{O}(1)$ coefficients of the 3-2 entries of $Y_{u}$ and $y_{u}$. The next benchmark point, "MFV-ct", features a large stop-scharm mixing at a higher messenger scale $M=10^{12} \mathrm{GeV}$, while keeping $F / M=2.7 \times 10^{5} \mathrm{GeV}$ fixed. At the messenger scale, $\left(y_{u}\right)_{32}=0.56$, and $\left(y_{u}\right)_{33}=1$. The resulting spectrum is shown in figure 4 . Here, the Higgs mass is $125 \mathrm{GeV}$. Again, the stop LR mixing, $\left|X_{t}\right| \sim 2.8 \mathrm{TeV}$ gives a large contribution to this mass. The lightest squark, at $1340 \mathrm{GeV}$, is an admixture of the R-stop and R-scharm. 
The L-stop and L-sbottom are heavy at $\sim 3.1 \mathrm{TeV}$, while other squarks are between $2.2-$ $2.5 \mathrm{TeV}$. Due to the high messenger scale, the bino NLSP is long-lived with a lifetime of $\tau_{\tilde{B}} \sim 0.3 \mathrm{~s}$, and does not decay inside the detector. Note that because of the large stopscharm mixing, the lightest squark can decay through either a third- or a second-generation quark, thus reducing the sensitivity of stop searches [26].

These examples will be probed by the standard LHC supersymmetry searches, including pair-production of gluinos, squarks, electroweakinos, and stops. Generally, searches for gluinos using simplified decay chains $\tilde{g} \rightarrow q q \tilde{\chi}_{0}^{1}$ have bounds of about $2.3 \mathrm{TeV}$ at the highluminosity LHC $[50,51]$; in the FGM scenarios, these bounds are weakened by the more complicated cascade decays. Searches for stop pair-production are particularly relevant for figure 3 because of the small stop mass $[52,53]$. If the bino decays to a $Z$ and a gravitino, and both the $W$ from the top decay and the $Z$ decay hadronically, the multi-jet plus missing energy searches are also useful $[54,55]$. In the spectrum of figure 4 , the lightest squark decays into either a second- or third-generation quark and the signal strengths of stop searches and jet plus missing energy squark searches are both reduced. Finally, searches for direct production of electroweakinos are also relevant for bino and wino masses in this range $[56,57]$.

\section{Non-MFV models}

We now turn to examples with large mass splittings between the first- and secondgeneration squarks. As explained in section 1, this requires an alignment of the down quark and squark mass matrices. To achieve this, we use a $\mathrm{U}(1) \times \mathrm{U}(1)$ horizontal symmetry. We consider three types of examples that realize unusual squark mass hierarchies [24]. These examples differ only in the horizontal charges of the messenger fields. The Higgs Yukawa coupling textures are thus the same in the three models, but the matter-messenger coupling matrices are different. Our main focus here is the masses and mixings of the firstand second-generation squarks. Thus, we do not insist on a $125 \mathrm{GeV}$ Higgs. In models with a lower Higgs mass, as is the case in our first example, some additional mechanism is required to raise the Higgs mass, such as the addition of the NMSSM singlet [5].

We choose the quark $\mathrm{U}(1) \times \mathrm{U}(1)$ charges to be,

$$
\begin{array}{lll}
Q_{1}(3,0), & Q_{2}(0,2), & Q_{3}(0,0), \\
u_{1}(-3,6), & u_{2}(1,0), & u_{3}(0,0), \\
d_{1}(-1,4), & d_{2}(4,-2), & d_{3}(0,2) .
\end{array}
$$

We assume that each $\mathrm{U}(1)$ is broken by a spurion of charge -1 and size $\lambda \sim 0.2$. These charges give rise to the following structures for the SM Yukawas, up to $\mathcal{O}(1)$ coefficients

$$
Y_{u} \sim\left(\begin{array}{ccc}
\lambda^{6} & \lambda^{4} & \lambda^{3} \\
0 & \lambda^{3} & \lambda^{2} \\
0 & \lambda & 1
\end{array}\right), \quad Y_{d} \sim\left(\begin{array}{ccc}
\lambda^{6} & 0 & \lambda^{5} \\
0 & \lambda^{4} & \lambda^{4} \\
0 & 0 & \lambda^{2}
\end{array}\right)
$$


This particular structure for $Y_{d}$ was shown in [58] to be the only pattern that can provide alignment in models with horizontal abelian symmetries. ${ }^{2}$ The diagonalizing matrices are then

$$
\begin{aligned}
V_{u L} \sim\left(\begin{array}{ccc}
1 & \lambda & \lambda^{3} \\
\lambda & 1 & \lambda^{2} \\
\lambda^{3} & \lambda^{2} & 1
\end{array}\right), & V_{u R} \sim\left(\begin{array}{ccc}
1 & \lambda^{4} & \lambda^{5} \\
\lambda^{4} & 1 & \lambda \\
\lambda^{9} & \lambda & 1
\end{array}\right), \\
V_{d L} \sim\left(\begin{array}{ccc}
1 & \lambda^{5} & \lambda^{3} \\
\lambda^{5} & 1 & \lambda^{2} \\
\lambda^{3} & \lambda^{2} & 1
\end{array}\right), & V_{d R} \sim\left(\begin{array}{ccc}
1 & \lambda^{7} & \lambda^{7} \\
\lambda^{7} & 1 & \lambda^{4} \\
\lambda^{7} & \lambda^{4} & 1
\end{array}\right),
\end{aligned}
$$

which give the correct CKM texture. In the following subsections, the single $\mathcal{O}(1)$ entry in $y_{u}$ will be denoted by $y$.

\subsection{Large $\left(y_{u}\right)_{11}$ : light up squark}

For our first example, we take the messenger field to carry a horizontal charge of

$$
\bar{D}(0,-6) \text {. }
$$

The messenger-Yukawa matrix is therefore

$$
y_{u} \sim\left(\begin{array}{lll}
y & 0 & 0 \\
0 & 0 & 0 \\
0 & 0 & 0
\end{array}\right) .
$$

The squark mass matrices are schematically given by eq. (2.11), with $W_{u L} \sim W_{u R} \sim$ $1_{3 \times 3}$; the only source of mixing is the fermion masses. In particular, to a good approximation, the only 1-2 mixing is in the L-up sector, as is generically the case with alignment in the down sector, and the only $2-3$ mixing is in the R-up sector; both are $\mathcal{O}(\lambda)$. The new coupling mainly affects the up and down squark masses, and can increase or decrease them depending on the value of $F / M^{2}$. The one-loop contributions are negative, while the two-loop contributions can have either sign. Since there is no large A-term, the Higgs mass requires extra ingredients, unless the stops are heavy.

Since $y$ is $\mathcal{O}(1)$, the dominant source of flavor violation is $\left(\delta_{12}^{u}\right)_{L L} \sim O(\lambda)$, which can be consistent with flavor bounds for certain choices of the model parameters. However, this estimate applies only to the absolute value of $\left(\delta_{12}^{u}\right)_{L L}$. The contribution to CP-violating processes is further suppressed, since $y$ only enters the soft masses as $|y|^{2}$. CP violation therefore originates solely from the SM Yukawas,

$$
\operatorname{Im}\left(\delta_{12}^{u}\right)_{L L} \sim \operatorname{Im}\left(V_{u L} y_{u}^{\text {diag }} y_{u}^{\text {diag }}{ }^{\dagger} V_{u L}^{\dagger}\right)_{12}=|y|^{2}\left(V_{u L}\right)_{11} \operatorname{Im}\left(V_{u L}{ }^{*}\right)_{21} \sim \lambda^{5},
$$

since $V_{u R} \sim V_{\mathrm{CKM}}$ in the 1-2 block. The imaginary part of $\left(\delta_{12}^{u}\right)_{R R}$ also originates from the SM Yukawas, but in this case, its source is $V_{u R}$,

$$
\operatorname{Im}\left(\delta_{12}^{u}\right)_{R R} \sim \operatorname{Im}\left(V_{u R} y_{u}^{\text {diag }^{\dagger}} y_{u}^{\operatorname{diag}} V_{u R}{ }^{\dagger}\right)_{12}=|y|^{2}\left(V_{u R}\right)_{11} \operatorname{Im}\left(V_{u R}{ }^{*}\right)_{21} .
$$

\footnotetext{
${ }^{2}$ This choice of charges is different from the choice of [24], which gave a zero $V_{t d}$. While the main results of [24] still hold, some details of the models are modified with this choice.
} 


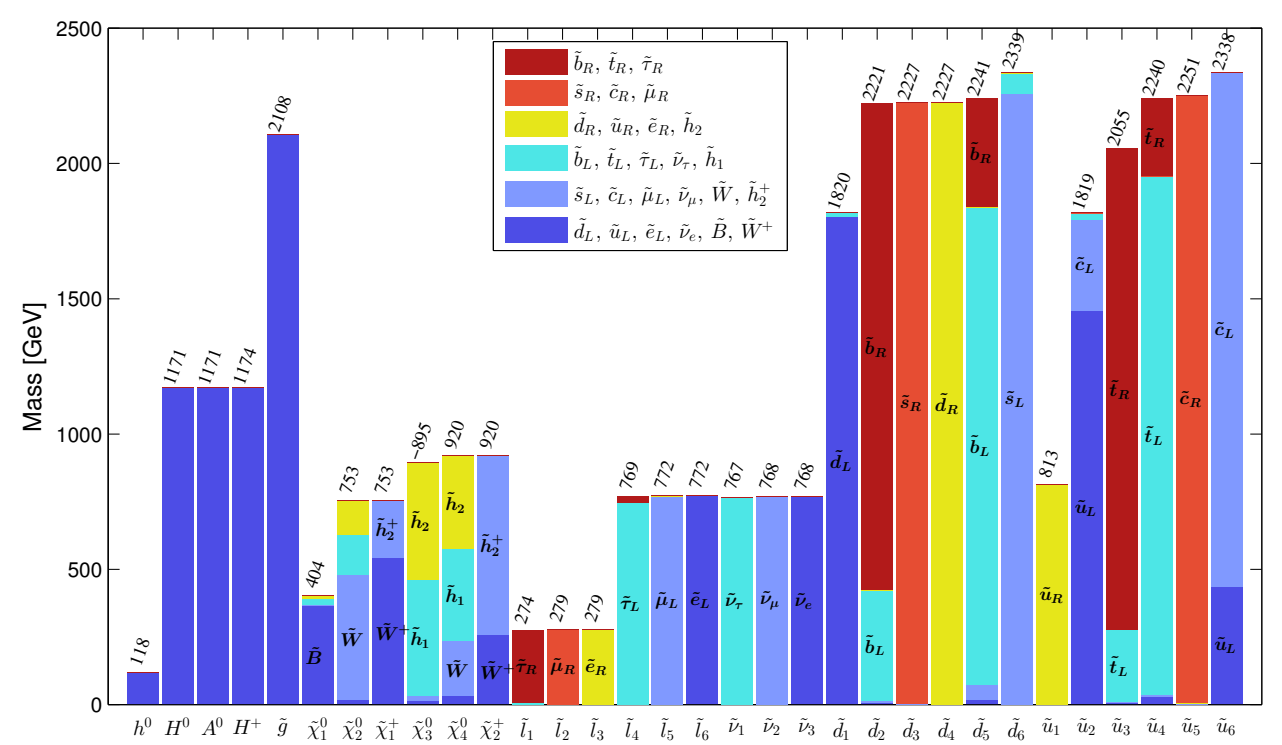

Figure 5. Spectrum for BP U11 with $N=2$, large $\left(y_{u}\right)_{11}\left(\Lambda=10^{16} \mathrm{GeV}\right)=0.4$, and scales $M=6.0 \times 10^{5} \mathrm{GeV}, F / M=1.49 \times 10^{5} \mathrm{GeV}$. Mixings are shown for sfermion and gaugino mass eigenstates. In this model, we find $\mu=885 \mathrm{GeV}$ and $\left(A_{u}\right)_{33}=-532 \mathrm{GeV}$.

Thus, $\left|\left(\delta_{12}^{u}\right)_{R R}\right| \sim \lambda^{4}$, and is very small. Still, $\sqrt{\operatorname{Im}\left(\left(\delta_{12}^{u}\right)_{L L}\left(\delta_{12}^{u}\right)_{R R}\right)}$ may be above the bound of table 1 by a factor of a few, and viable models may require some suppression of the phases in the 1-2 block of $V_{u R}$. In the examples below, the required suppression is $\sim 0.4$.

Figure 5 shows the spectrum of a BP, "U11", with $N_{5}=2$. Here the gluino is at $2.1 \mathrm{TeV}$. The R-up squark is at $810 \mathrm{GeV}$, while all other squarks have masses between 1.8-2.3 TeV. Thus, there is a single light squark in the spectrum. The large squark mass splitting gives a negative (positive) contribution to the $\mathrm{R}(\mathrm{L})$-slepton masses through the running $[5,11,16]$ resulting in an R-stau NLSP with a decay length of $c \tau_{\tilde{\tau}} \geq 5.0 \mathrm{~mm}$, where the lower value is attained if the dominant source of supersymmetry breaking is the $F$-term of $X$. For a larger value of this parameter, the stau can become long-lived and detectable in searches for long-lived charged particles $[59,60]$.

\subsection{Large $\left(y_{u}\right)_{22}$ : light charm squark}

This example is qualitatively similar, with the large effects occurring in the secondgeneration squarks. We take the $\bar{D}$ messenger charges to be,

$$
\bar{D}(-1,-2)
$$

so that

$$
y_{u} \sim\left(\begin{array}{lll}
0 & 0 & 0 \\
0 & y & 0 \\
0 & 0 & 0
\end{array}\right) .
$$

The main effects are therefore on the charm and strange squark masses, and these can have either sign. 


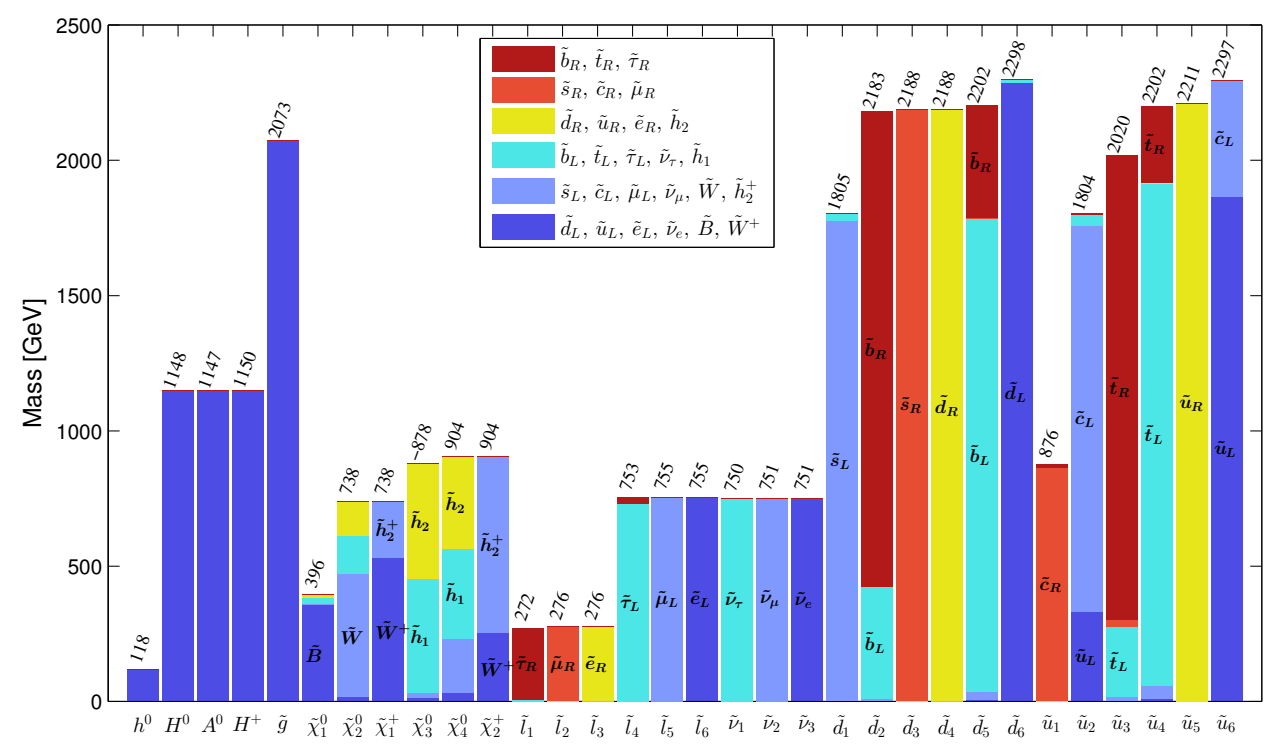

Figure 6. Spectrum for BP U22L with $N_{5}=2, M=5.7 \times 10^{5} \mathrm{GeV}, F / M=1.46 \times 10^{5} \mathrm{GeV}$, and $\left(y_{u}\right)_{22}\left(\Lambda=10^{16} \mathrm{GeV}\right)=0.7$. Mixings are shown for sfermion and gaugino mass eigenstates In this model, we find $\mu=869 \mathrm{GeV}$ and $A_{u}^{33}=-521 \mathrm{GeV}$.

We note that the horizontal charges also allow a small $O\left(\lambda^{3}\right)$ mixing between $H_{U}$ and $\bar{D}$ from the Kähler and superpotential, changing the Yukawa structure to (see appendix A)

$$
y_{u} \rightarrow\left(\begin{array}{ccc}
\sim 0 & \lambda^{7} & \lambda^{6} \\
0 & y & \lambda^{5} \\
0 & \lambda^{4} & \lambda^{3}
\end{array}\right)
$$

These corrections are small, and proportional to the SM Yukawas. Therefore the squarkquark mixings are again given by the SM flavor mixing matrices, with $W_{u L}=W_{u R} \sim 1_{3 \times 3}$, as in the previous example.

We show two examples in which the new contributions to the charm squark are negative, resulting in a single charm squark that is much lighter than the remaining squarks. The flavor-violating $\delta$ 's in these examples are similar to those of the previous example. In figure 6 we display the spectrum for BP "U22L" obtained for a messenger scale $M=5.7 \times 10^{5} \mathrm{GeV}$ and $N_{5}=2$. The R-scharm is at $870 \mathrm{GeV}$, the remaining squarks have masses above $1.8 \mathrm{TeV}$, and the gluino mass is around $2 \mathrm{TeV}$. Here and in the next BP U22, the large squark mass splitting feeds into the RGE's of the sleptons and creates a large splitting between the R- and L-sleptons, so that the R-stau is the NLSP, while the L-sleptons are all heavier than the winos. The stau NLSP decays to a tau and gravitino, with a lower bound on the decay length of $c \tau_{\tilde{\tau}} \geq 0.05 \mathrm{~mm}$; for much longer lifetimes, both examples would be excluded by long-lived stau searches. The R-sleptons have approximately degenerate masses of $\sim 270 \mathrm{GeV}$, which currently lie above searches using direct production [61, 62]. SUSY searches with multilepton final states can also be useful to look for cascade decays of the R-up squark [63]. In contrast to minimal GMSB spectra, the 


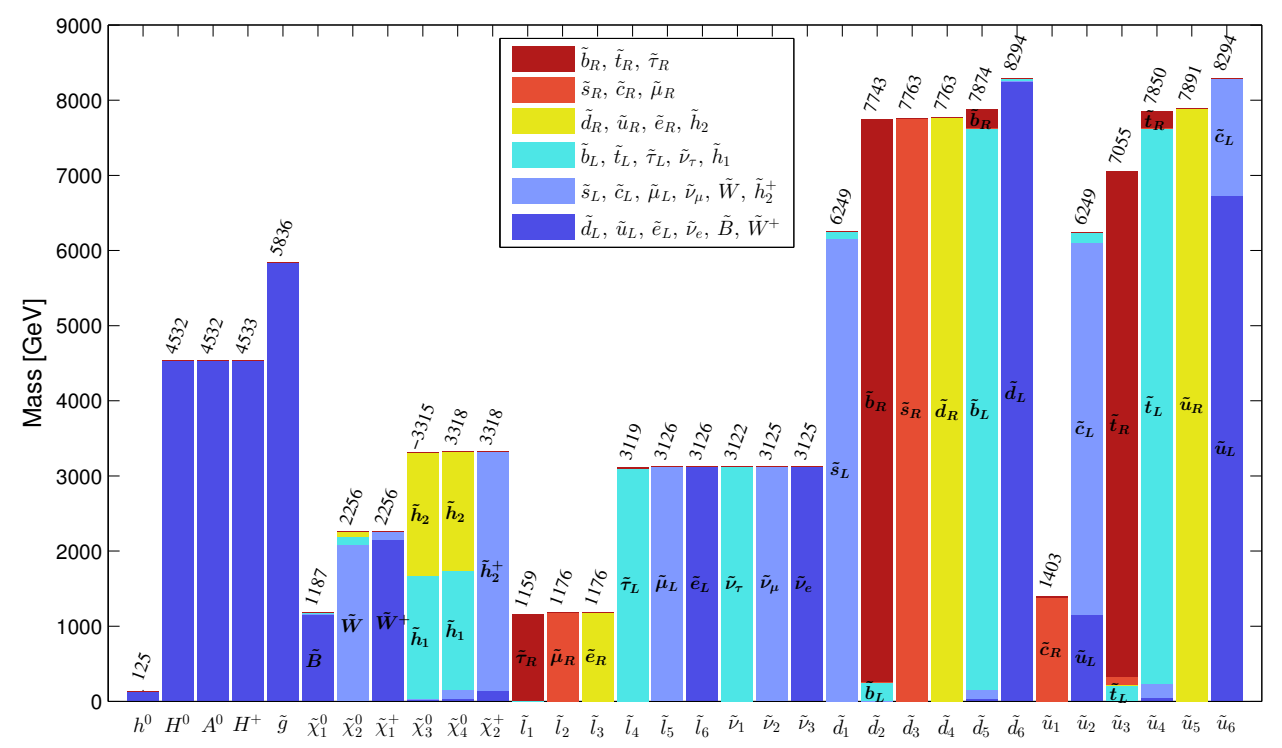

Figure 7. Spectrum for BP U22 with large $\left(y_{u}\right)_{22}\left(\Lambda=10^{16} \mathrm{GeV}\right)=0.73$ and scales $M=10^{7} \mathrm{GeV}$, $F / M=8.6 \times 10^{5} \mathrm{GeV}$. Mixings are shown for sfermion and gaugino mass eigenstates. In this model, we find $\mu=3280 \mathrm{GeV}$ and $\left(A_{u}\right)_{33}=-1540 \mathrm{GeV}$.

quarks are almost exclusively of charm flavor, so charm tagging will play an important role in these cases [64].

The second BP "U22" demonstrates that a large Higgs mass can be obtained in these models from very heavy stops, while a single squark may still be within LHC reach. The stops in this example are at $7 \mathrm{TeV}$. Since only a single squark flavor is significantly affected by the messenger coupling, most of the remaining squarks are around $7 \mathrm{TeV}$ too, with the exception of the R-scharm at $1.4 \mathrm{TeV}$. The spectrum is shown in figure 7 , for $M=10^{7} \mathrm{GeV}$, $y\left(\Lambda=10^{16} \mathrm{GeV}\right)=0.73$, and $F / M=8.6 \times 10^{5} \mathrm{GeV}$.

This spectrum has a long-lived NLSP stau with $c \tau_{\tilde{\tau}} \geq 0.4 \mathrm{~mm}$, which is mainly produced at the LHC in the cascade decay of $\tilde{u}_{1}$. Therefore, we expect two long-lived charged particles accompanying two quarks.

\subsection{Large $\left(y_{u}\right)_{32}$ : light top and/or charm squark}

As a final example, we consider models with a large 3-2 entry in the messenger coupling matrix. This leads to a large stop A-term, which allows for a $125 \mathrm{GeV}$ Higgs with superpartners accessible at the LHC. At the same time, there are large modification of the L-stop, L-sbottom and R-scharm masses, and in some examples, a large stop-scharm mixing.

To obtain a large $\left(y_{u}\right)_{32}$ we take the messenger horizontal charges to be

$$
\bar{D}(-1,0) \text {, }
$$

which yields the messenger Yukawa matrix

$$
y_{u} \sim\left(\begin{array}{ccc}
0 & \lambda^{3} & \lambda^{2} \\
0 & \lambda^{2} & 0 \\
0 & y & 0
\end{array}\right) .
$$


As above, $y$ is an $\mathcal{O}(1)$ coefficient which we display explicitly since it is the dominant entry in the $y_{u}$ matrix.

As discussed in appendix $\mathrm{A}$, these charges also allow a $\lambda X D H_{U}$ term in the superpotential, and a $\lambda \bar{D}^{\dagger} H_{U}$ term in the Kähler potential. Therefore, the Yukawa matrices may in principle be modified as $Y_{u} \rightarrow Y_{u}+\lambda y_{u}$ and $y_{u} \rightarrow y_{u}+\lambda Y_{u}$. This only affects the texture of $y_{u}$, with

$$
y_{u} \rightarrow\left(\begin{array}{ccc}
\lambda^{7} & \lambda^{3} & \lambda^{2} \\
0 & \lambda^{2} & \lambda^{3} \\
0 & y & \lambda
\end{array}\right)
$$

Since the messenger coupling has an $\mathcal{O}(1) 3-2$ entry, mixed terms involving both $y_{u}$ and the SM Yukawas are important in these models. Examining the structure of the soft terms in eq. (B.16), one gets,

$$
\begin{aligned}
& \delta \tilde{m}_{q}^{2} \sim-\frac{1}{(4 \pi)^{2}} \frac{1}{6}\left(\begin{array}{ccc}
\lambda^{4} & \lambda^{5} & y \lambda^{3} \\
\lambda^{5} & \lambda^{4} & y \lambda^{2} \\
y \lambda^{3} & y \lambda^{2} & |y|^{2}
\end{array}\right) \frac{F^{4}}{M^{6}} \\
& +\frac{1}{(4 \pi)^{4}}\left(\begin{array}{ccc}
\left(3|y|^{2}+2-G\right) \lambda^{4} & \left(6|y|^{2}+3 y+2-G\right) \lambda^{5} & \left(6 y^{3}+5 y-G y\right) \lambda^{3} \\
\left(6|y|^{2}+3 y+2-G\right) \lambda^{5} & \left(6|y|^{2}-G\right) \lambda^{4} & \left(6 y^{3}-G y\right) \lambda^{2} \\
\left(6 y^{3}+5 y-G y\right) \lambda^{3} & \left(6 y^{3}-G y\right) \lambda^{2} & \left(6|y|^{4}-G|y|^{2}\right)
\end{array}\right)\left|\frac{F}{M}\right|^{2} \\
& \delta \tilde{m}_{u_{R}}^{2} \sim-\frac{1}{(4 \pi)^{2}} \frac{1}{3}\left(\begin{array}{ccc}
0 & 0 & 0 \\
0 & |y|^{2} & -y \lambda^{3} \\
0 & -y \lambda^{3} & \lambda^{4}
\end{array}\right) \frac{F^{4}}{M^{6}} \\
& +\frac{1}{(4 \pi)^{4}}\left(\begin{array}{ccc}
0 & 0 & 0 \\
0 & (2-2 G)|y|^{2}+12|y|^{4} & 4|y|^{2} \lambda \\
0 & 4|y|^{2} \lambda & -2|y|^{2}
\end{array}\right)\left|\frac{F}{M}\right|^{2} \\
& \delta \tilde{m}_{d_{R}}^{2} \sim-\frac{1}{(4 \pi)^{2}} 2\left(\begin{array}{ccc}
0 & 0 & 0 \\
0 & 0 & 0 \\
0 & 0 & |y|^{2} \lambda^{4}
\end{array}\right)\left|\frac{F}{M}\right|^{2}
\end{aligned}
$$

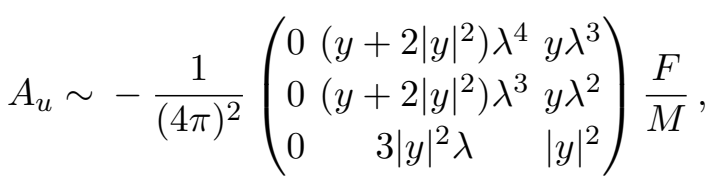

$$
\begin{aligned}
& A_{d} \sim-\frac{1}{(4 \pi)^{2}}\left(\begin{array}{ccc}
0 & 0 & y \lambda^{5} \\
0 & 0 & y \lambda^{4} \\
0 & 0 & |y|^{2} \lambda^{2}
\end{array}\right) \frac{F}{M},
\end{aligned}
$$

where we neglected $\mathcal{O}\left(\lambda^{6}\right)$ terms. Here $G=\frac{16}{3} g_{3}^{2}+3 g_{2}^{2}+\frac{13}{15} g_{1}^{2}$ is between 5 and 10 , depending on the messenger scale. Recall that, in addition to $G$ and the explicit numerical factors that appear above, there are other unknown numerical factors coming from the Yukawa 


\begin{tabular}{|cc|ccc|}
\hline$q$ & $i j$ & $\left|\left(\delta_{i j}^{q}\right)_{L L}\right|$ & $\left|\left(\delta_{i j}^{q}\right)_{R R}\right|$ & $\sqrt{\left|\left(\delta_{i j}^{q}\right)_{L L}\right|\left|\left(\delta_{i j}^{q}\right)_{R R}\right|}$ \\
\hline$d$ & 12 & $\lambda^{5}$ & - & - \\
$u$ & 12 & $\lambda^{5}$ & $\lambda^{4}$ & $\lambda^{4.5}$ \\
$d$ & 23 & $\lambda^{2}$ & $\lambda^{8}$ & $\lambda^{5}$ \\
\hline
\end{tabular}

Table 3. Parametric estimates of $\left(\delta_{i j}^{q}\right)_{\mathrm{MM}}$ in non-MFV models with large $\left(y_{u}\right)_{32}$. Omitted entries are negligible.

couplings themselves. The quark-squark gluino mixings are then given by,

$$
\begin{aligned}
K_{u}^{L} \sim\left(\begin{array}{ccc}
1 & \lambda & \lambda^{3} \\
\lambda & 1 & \lambda^{2} \\
\lambda^{3} & \lambda^{2} & 1
\end{array}\right), & K_{u}^{R} \sim\left(\begin{array}{ccc}
1 & \lambda^{4} & \lambda^{5} \\
\lambda^{4} & 1 & \lambda \\
0 & \lambda & 1
\end{array}\right), \\
K_{d}^{L} \sim\left(\begin{array}{ccc}
1 & \lambda & \lambda^{3} \\
\lambda & 1 & \lambda^{2} \\
\lambda^{3} & \lambda^{2} & 1
\end{array}\right), & K_{d}^{R} \sim\left(\begin{array}{ccc}
1 & \lambda^{3} & \lambda^{7} \\
\lambda^{3} & 1 & \lambda^{4} \\
\lambda^{7} & \lambda^{4} & 1
\end{array}\right),
\end{aligned}
$$

These models then have the following general features:

1. A large negative contribution to the R-stop mass $\left(\tilde{m}_{u_{R}}^{2}\right)_{33}$.

2. Large contributions to the R-scharm mass, $\left(\tilde{m}_{u_{R}}^{2}\right)_{22}$. Here the one-loop contribution is negative, and the two-loop contribution can have either sign.

3. Large contributions to the L-stop and L-sbottom masses, $\left(\tilde{m}_{q}^{2}\right)_{33}$. Here the one-loop contribution is negative, while the two-loop contribution can have either sign.

4. An $\mathcal{O}(\lambda) \mathrm{R}$ stop-scharm mixing, $\left(\tilde{m}_{u_{R}}^{2}\right)_{32}$.

5. A large stop A-term $\left(A_{u}\right)_{33}$.

The parametric estimates for the relevant flavor-violating quantities in these models are collected in table 3 . These are clearly compatible with current experimental bounds. In particular, $\left(\delta_{12}^{q}\right)_{L L}$ is small mainly because of the $\mathcal{O}\left(\lambda^{4}\right)$ mass splitting in the L-squarks (accompanied by an $\mathcal{O}(\lambda)$ mixing). In contrast, there is an $\mathcal{O}(1)$ mass splitting between the R-charm and R-up squarks, but the mixing between them is $\mathcal{O}\left(\lambda^{4}\right)$.

As in the MFV-like models, since the messenger couplings to the third generation are large here, the running between the flavor scale $\Lambda$ and the messenger scale $M$ can modify both the messenger couplings and the SM Yukawa couplings. In figure 8 we show $\left(Y_{u}\right)_{33}(M)$ and $\left(y_{u}\right)_{32}(M)$ as a function of $\left(y_{u}\right)_{32}(\Lambda)=y$, for various values of $\left(Y_{u}\right)_{33}(\Lambda)$. As it decreases in the running, $\left(Y_{u}\right)_{33}$ requires a large boundary value at $\Lambda$. Furthermore, for a wide range of boundary values at $\Lambda,\left(y_{u}\right)_{32}$ flows to values near 1 at the messenger scale. Thus the size of the new messenger coupling is limited by the running. 

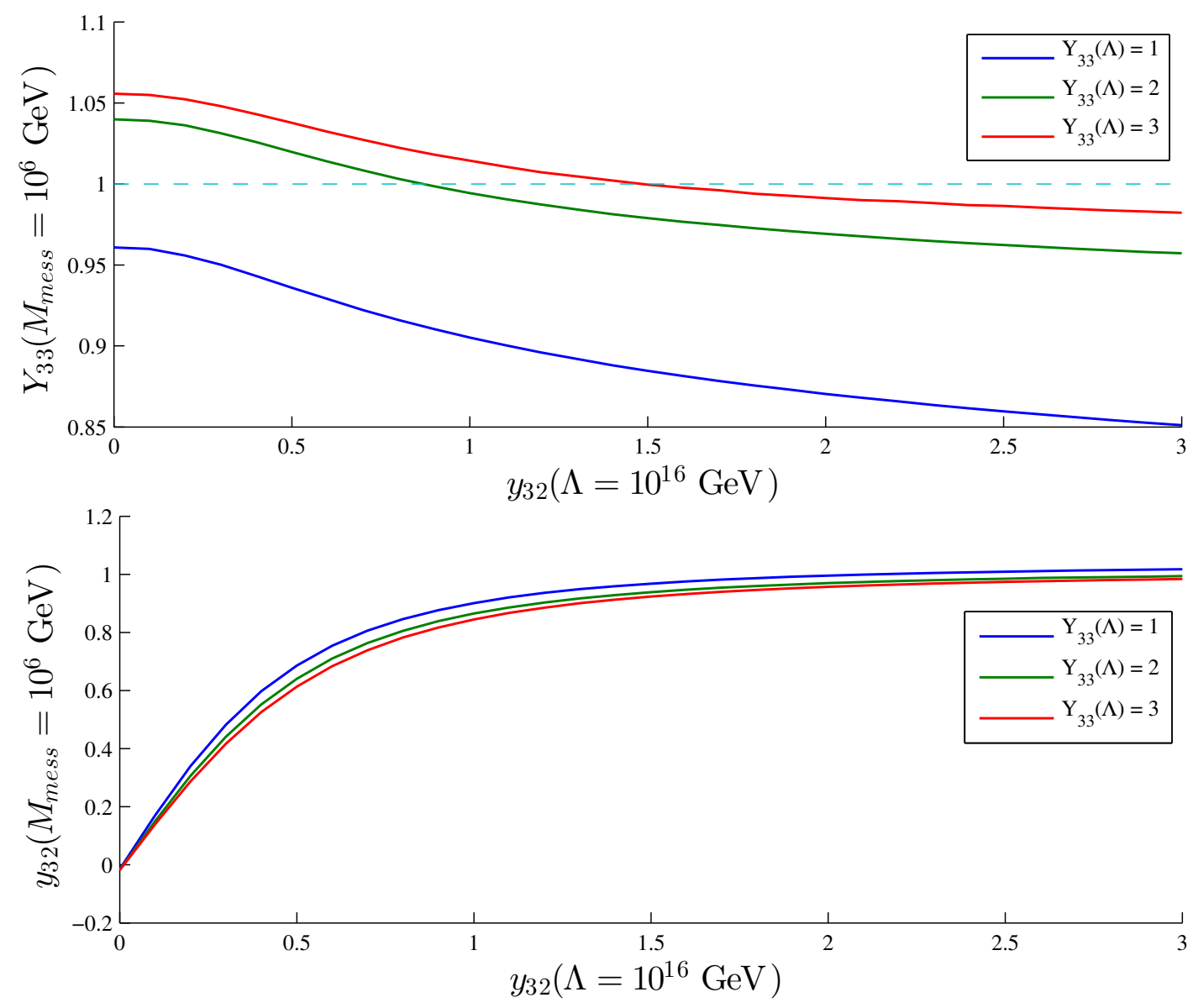

Figure 8. $\left(y_{u}\right)_{32}$ and $\left(Y_{u}\right)_{33}$ at the messenger scale $M=10^{6} \mathrm{GeV}$ as functions of $\left(y_{u}\right)_{32}(\Lambda)=y$ for various $\mathcal{O}(1)$ values of $\left(Y_{u}\right)_{33}(\Lambda)$.

Three spectra of benchmark points using this model are shown in figures 9-11. All of these have a Higgs mass of $125-126 \mathrm{GeV}$, partly driven by the large stop mixing, which in turn comes from the large stop A-term coupled with, in some cases, a negative contribution to the stop soft masses from $\left(y_{u}\right)_{32}$. We have taken the flavor scale $\Lambda=10^{16} \mathrm{GeV}$ in these examples.

The first BP "U32-c" features a very heavy spectrum, with a single squark below the $\mathrm{TeV}$ scale. Specifically, the R-scharm is at $815 \mathrm{GeV}$, the stops and L-sbottom are between 2.7-3 TeV, and all other colored superpartners are above $4 \mathrm{TeV}$. This spectrum, shown in figure 9 is obtained for $M=3.0 \times 10^{6} \mathrm{GeV},\left(y_{u}\right)_{32}(\Lambda)=0.89, F / M=5.25 \times 10^{5} \mathrm{GeV}$, where the one- and two-loop contributions are comparable. The NLSP is the stau; it promptly decays to a tau and gravitino with the lower bound $c \tau_{\tilde{\tau}}>5 \mathrm{~mm}$.

In the next spectrum for BP "U32-t", shown in figure 10, the two-loop contribution is dominant for the parameter choices $M=10^{7} \mathrm{GeV}, F / M=3.52 \times 10^{5} \mathrm{GeV}$, and $\left(y_{u}\right)_{32}(\Lambda)=1.57$. The gluino is at $2.5 \mathrm{TeV}$ and all squarks, with the exception of the R-stop, are between $3-4 \mathrm{TeV}$. Due to the negative contribution from $\left(y_{u}\right)_{32}$, the mass of 


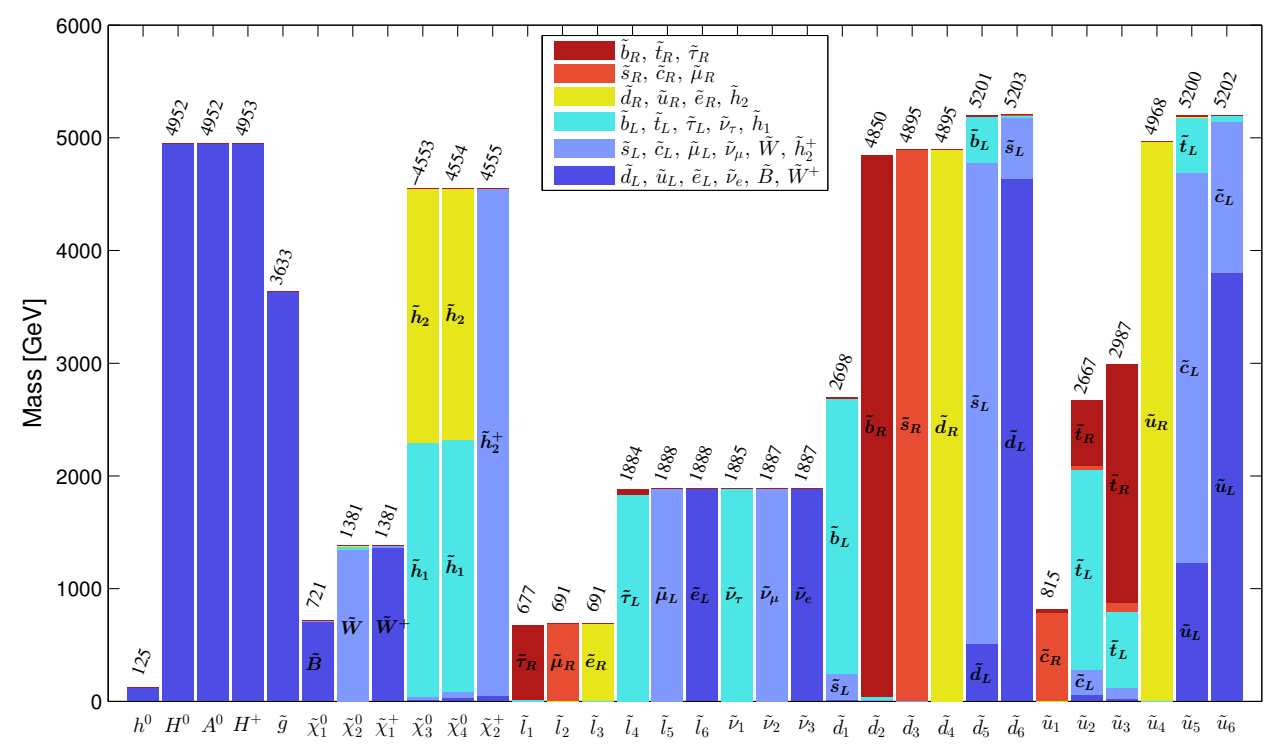

Figure 9. Spectrum for BP U32-c with large $\left(y_{u}\right)_{32}\left(\Lambda=10^{16} \mathrm{GeV}\right)=0.89$ and scales $M=$ $3.0 \times 10^{6} \mathrm{GeV}, F / M=5.25 \times 10^{5} \mathrm{GeV}$. Mixings are shown for sfermion and gaugino mass eigenstates. In this model, we find $\mu=4580 \mathrm{GeV}$ and $\left(A_{u}\right)_{33}=-2880 \mathrm{GeV}$.

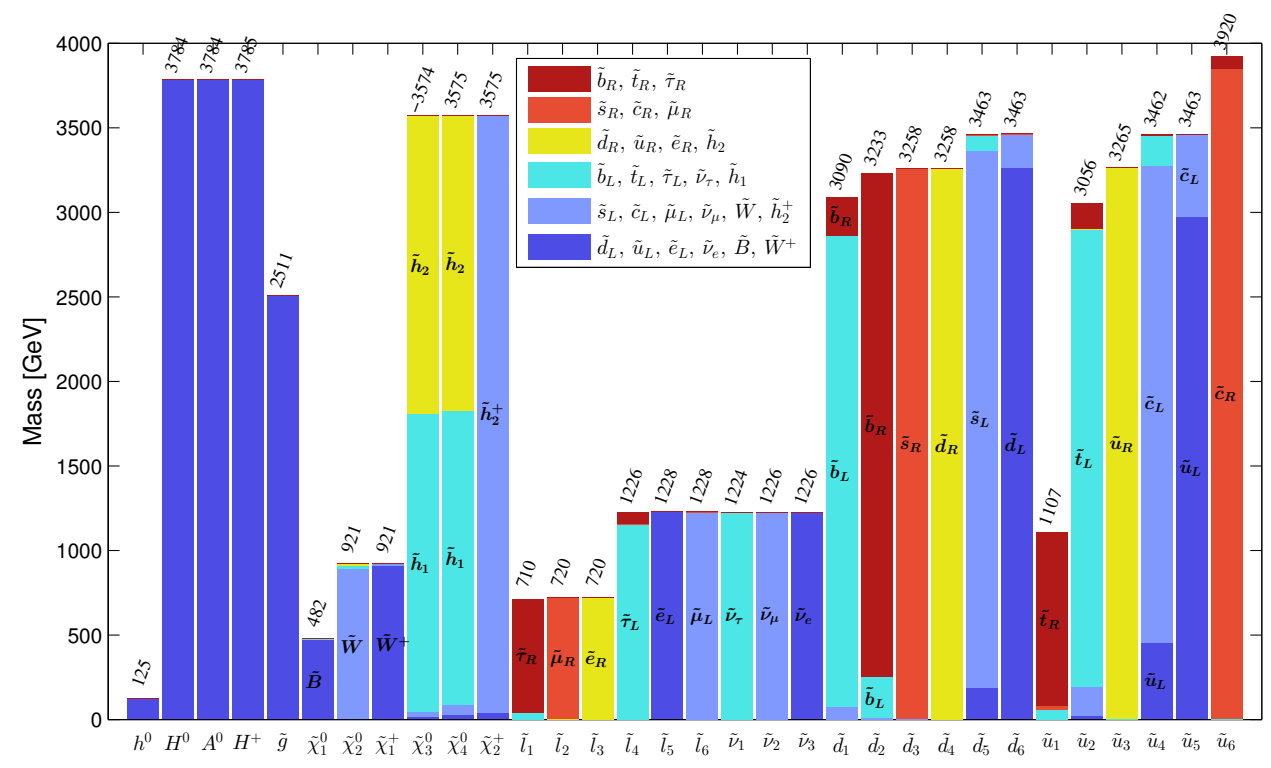

Figure 10. Spectrum for BP U32-t with $M=10^{7} \mathrm{GeV}, F / M=3.52 \times 10^{5} \mathrm{GeV}$, and $y_{u}^{32}(\Lambda=$ $\left.10^{16} \mathrm{GeV}\right)=1.57$. Mixings are shown for sfermion and gaugino mass eigenstates. In this model, we find $\mu=3580 \mathrm{GeV}$ and $A_{u}^{33}=-2360 \mathrm{GeV}$.

the R-stop is much lower at $1.1 \mathrm{TeV}$. The NLSP in this model is the bino, with a lower bound on the decay length of $c \tau_{\tilde{B}}>4 \mathrm{~mm}$.

Finally, the last BP "U32-ct", shown in figure 11, has a lighter spectrum, but features a mixed scharm-stop as the lightest squark around $1.3 \mathrm{TeV}$, making stop searches relying 


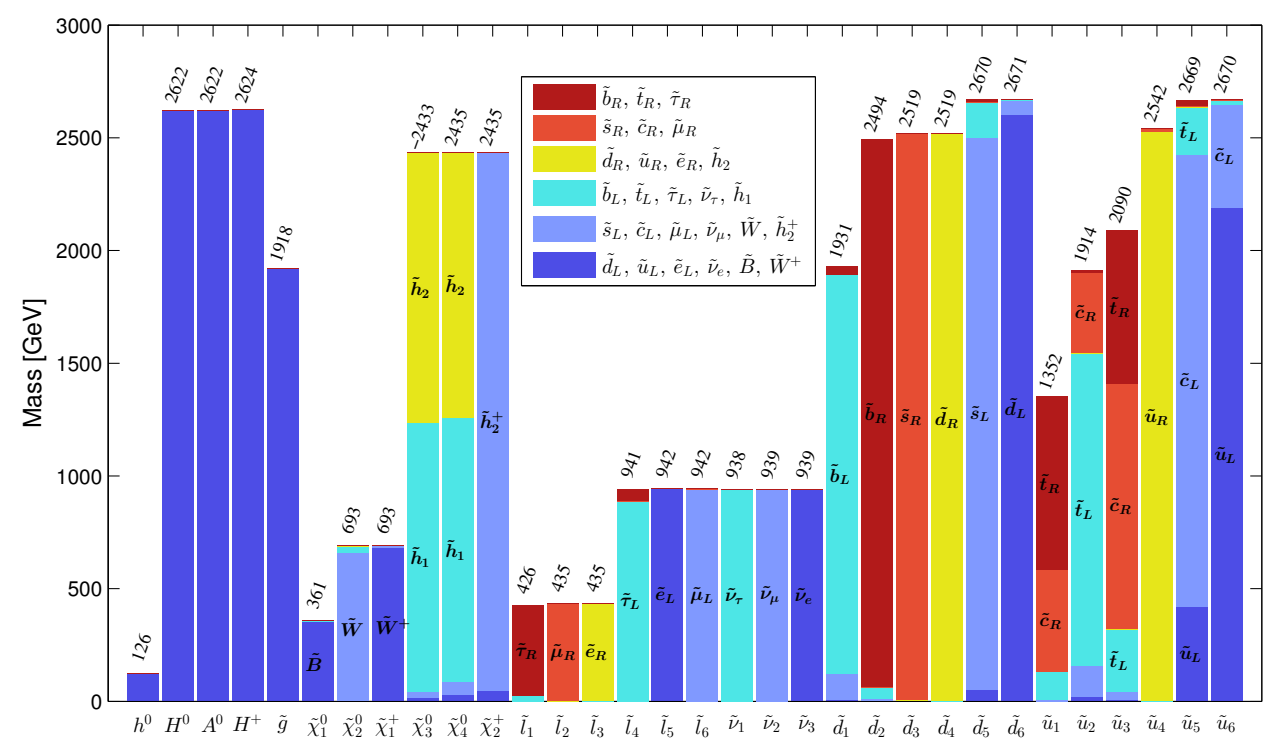

Figure 11. Spectrum for BP U32-ct with large $\left(y_{u}\right)_{32}\left(\Lambda=10^{16} \mathrm{GeV}\right)=1.21,\left(y_{u}\right)_{33}(\Lambda=$ $\left.10^{16} \mathrm{GeV}\right)=0.6$ and scales $M=3.0 \times 10^{6} \mathrm{GeV}, F / M=2.66 \times 10^{5} \mathrm{GeV}$. Mixings are shown for sfermion and gaugino mass eigenstates. In this model, we find $\mu=2450 \mathrm{GeV}$ and $\left(A_{u}\right)_{33}=-1460 \mathrm{GeV}$.

on decays to third-generation quarks even more challenging. The lower bound on the decay length of the bino NLSP is $c \tau_{\tilde{B}}>0.04 \mathrm{~mm}$.

The lightest squark and NLSP of these models will largely determine their collider signatures. In terms of relevant searches, each of these examples has overlap with previous examples: figure 9 with figure 7 (light R-scharm and R-stau NLSP), figure 10 with figure 3 (light R-stop and bino NLSP), and figure 11 with figure 4 (light mixed R stop-scharm and bino NLSP). The NLSP lifetimes for figures 9-10 are longer due to the larger values of $F$. Comparing the flavour bounds in the 1-2 up sector listed in table 5, we see that the constraints from observables in D mesons can be used to distinguish these pairs of spectra.

Note that the models discussed in this subsection have a non-trivial A-term structure, with a potentially large $\left(A_{u}\right)_{32}$. Furthermore, some of the examples have a large stopscharm mixing. Both of these features may affect the calculation of the Higgs mass by a few GeV [43-46]. These effects are not taken into account in SOFTSUSY.

\section{Conclusions}

We presented examples of flavored gauge mediation with interesting and unusual squark spectra that are nonetheless consistent with low-energy constraints.

These examples are derived from fully calculable models. Specifically, the soft terms are generated by messenger-field loops, and the size of the different superpotential couplings - both the SM Yukawas and the messenger Yukawas - are determined by a flavor symmetry. In many cases, the structure of the soft terms leads to stronger suppression of 
low-energy flavor-violating processes, compared to naive estimates which are based on the flavor symmetry alone.

The smallness of observed flavor-violating processes has long been viewed as a major constraint on superpartner flavor. The above discussion suggests that this viewpoint is perhaps too restrictive, and is the consequence of considering general ansatze for supersymmetry and for flavor, rather than concrete models.

\section{Acknowledgments}

We thank Iftah Galon, Mark Goodsell, Sven Heinemeyer, Yossi Nir, Gilad Perez, and Ofri Telem for discussions. Research supported by the Israel Science Foundation (Grant No. 720/15), by the United-States-Israel Binational Science Foundation (BSF) (Grant No. 2014397), and by the ICORE Program of the Israel Planning and Budgeting Committee (Grant No. 1937/12).

\section{A FGM superpotential and symmetries}

We summarize some details of the construction of the models from [1, 24]. The form of the superpotential in eq. (2.1) can be enforced by various choices of symmetries. Clearly, the messengers are taken to have the same R-parity as the Higgs fields. One possibility (which is not necessarily the most economical one) is to introduce a $\mathrm{U}(1)_{X}$ under which $X$ has charge 1 , and $T_{I}, D_{I \neq 2}, \bar{D}_{2}$ have charge -1 , as well as a messenger parity for the messenger fields with $I>2{ }^{3}$ This still allows for the superpotential terms $X D_{1} H_{U}$ and $X \bar{D}_{2} H_{D}$. We return to these below.

To allow only up-type messenger couplings, we take instead $T_{I}$ and $D_{I}$ to have $\mathrm{U}(1)_{X}$ charge -1 . The only term we can add to the superpotential in eq. (2.1) is $\gamma X D_{1} H_{U}$, where $\gamma$ is some coefficient. In the MFV-like models, where the flavor symmetry does not distinguish between $\bar{D}_{1}$ and $H_{U}, \gamma \sim 1$, and we can redefine the messenger field as the combination of $H_{U}$ and $\bar{D}_{1}$ that couples to $X$, thus eliminating the term $\gamma X D_{1} H_{U}$.

In the non-MFV models of this paper, $\gamma$ is given by some power of $\lambda$. In addition, the Kähler potential contains mixing terms such as $\delta \bar{D}^{\dagger} H_{U}$ with $\delta \sim \gamma$. Redefining and rotating the fields to get a canonical Kähler potential and to eliminate the superpotential term $X D_{1} H_{U}$, one obtains the superpotential in eq. (2.1) with $Y_{U} \rightarrow Y_{U}+\gamma y_{U}$, and $y_{U} \rightarrow y_{U}+\epsilon Y_{U}$, where $\epsilon \sim \gamma, \delta$. Alternatively, one can use the flavor symmetry to eliminate the superpotential mixing term altogether [1].

We also assume that the $\mu$ term is forbidden by an additional PQ symmetry. We will not discuss the origin of the $\mu$ term, although it is possible to embed this PQ symmetry in the flavor symmetry [1], and to generate a small "supersymmetric" $\mu$ term. ${ }^{4}$

\footnotetext{
${ }^{3}$ Motivated by the fact that full dynamical supersymmetry-breaking models that generate the required vacuum expectation values for $X$ often require an $X^{3}$ term [3], earlier FGM papers starting with [1] considered a $Z_{3}$ instead of a $\mathrm{U}(1)$.

${ }^{4}$ We note that many of our examples require only a small $B$ term which can be obtained radiatively from the $\mu$ term.
} 


\section{B Soft terms}

We first specify our conventions for the Yukawas and soft terms. We define the Lagrangian and the superpotential as

$$
\begin{aligned}
\mathcal{L} & =\int \mathrm{d}^{4} \theta K+\left(\int \mathrm{d}^{2} \theta W+\text { h.c. }\right), \\
W & =X\left(T_{I} \bar{T}_{I}+D_{I} \bar{D}_{I}\right)+y_{U i j} \bar{D}_{1} q_{i} u_{j}^{c}+Y_{U i j} H_{U} q_{i} u_{j}^{c}+Y_{D i j} H_{D} q_{i} d_{j}^{c}+Y_{L i j} H_{D} l_{i} e_{j}^{c},
\end{aligned}
$$

which yields the SM Yukawa interactions

$$
\mathcal{L} \supset-Y_{U i j} H_{U}\left(\psi_{q}\right)_{i}\left(\psi_{u^{c}}\right)_{j}
$$

etc., and the soft terms

$$
-\mathcal{L} \supset \tilde{m}_{q i j}^{2} \tilde{q}_{i}^{*} \tilde{q}_{j}+\tilde{m}_{l i j}^{2} \tilde{l}_{i}^{*} \tilde{l}_{j}+\tilde{m}_{u_{R} i j}^{2} \tilde{u}_{i}^{*} \tilde{u}_{j}+\tilde{m}_{d_{R} i j}^{2} \tilde{d}_{i}^{*} \tilde{d}_{j}+\cdots+A_{u i j} H_{U} \tilde{q}_{i} \tilde{u}_{j}^{*}+\cdots,
$$

where $\tilde{q}$ and $\psi_{q}\left(\tilde{u}^{*}\right.$ and $\left.\psi_{u^{c}}\right)$ are the scalar and fermion components of the superfield $q$ $\left(u^{c}\right)$. In this paper, following [16], we use the common notation for the SM Yukawas

$$
Y_{u}=\left(Y_{U}\right)^{*}, \quad Y_{d}=\left(Y_{D}\right)^{*}, \quad y_{u}=\left(y_{U}\right)^{*},
$$

which appears in the Lagrangian as (e.g., section 11 of [65]),

$$
\mathcal{L} \supset-Y_{u i j} H_{U}\left(\overline{\psi_{q}}\right)_{i}\left(\psi_{u}\right)_{j} .
$$

The leading order GMSB contributions to the soft masses are $[2,3,66]$

$$
\begin{aligned}
\tilde{M}_{i} & =\frac{g_{i}^{2}}{(4 \pi)^{2}} N_{5} \frac{F}{M} g(x), \\
\tilde{m}_{H_{U}}^{2} & =\frac{1}{(4 \pi)^{4}} 2 N_{5}\left(\frac{3}{4} g_{2}^{4}+\frac{3}{20} g_{1}^{4}\right)\left|\frac{F}{M}\right|^{2}, \\
\tilde{m}_{H_{D}}^{2} & =\frac{1}{(4 \pi)^{4}} 2 N_{5}\left(\frac{3}{4} g_{2}^{4}+\frac{3}{20} g_{1}^{4}\right)\left|\frac{F}{M}\right|^{2}, \\
\tilde{m}_{q}^{2} & =\frac{1}{(4 \pi)^{4}} 2 N_{5}\left(\frac{4}{3} g_{3}^{4}+\frac{3}{4} g_{2}^{4}+\frac{1}{60} g_{1}^{4}\right)\left|\frac{F}{M}\right|^{2} 1_{3 \times 3}, \\
\tilde{m}_{u_{R}}^{2} & =\frac{1}{(4 \pi)^{4}} 2 N_{5}\left(\frac{4}{3} g_{3}^{4}+g_{1}^{4} \frac{4}{15}\right)\left|\frac{F}{M}\right|^{2} 1_{3 \times 3}, \\
\tilde{m}_{d_{R}}^{2} & =\frac{1}{(4 \pi)^{4}} 2 N_{5}\left(\frac{4}{3} g_{3}^{4}+\frac{1}{15} g_{1}^{4}\right)\left|\frac{F}{M}\right|^{2} 1_{3 \times 3}, \\
\tilde{m}_{l}^{2} & =\frac{1}{(4 \pi)^{4}} 2 N_{5}\left(\frac{3}{4} g_{2}^{4}+\frac{3}{20} g_{1}^{4}\right)\left|\frac{F}{M}\right|^{2} 1_{3 \times 3}, \\
\tilde{m}_{e}^{2} & =\frac{1}{(4 \pi)^{4}} 2 N_{5}\left(\frac{3}{5} g_{1}^{4}\right)\left|\frac{F}{M}\right|^{2} 1_{3 \times 3},
\end{aligned}
$$


at the messenger scale, where $x=F / M^{2}$ and [67]

$$
g(x)=\frac{1}{x^{2}}[(1+x) \log (1+x)+(1-x) \log (1-x)]=1+\frac{x^{2}}{6}+O\left(x^{4}\right) .
$$

We did not include the corresponding term for sfermion masses [68] in our numerical calculations. This is for consistency of the two-loop messenger-matter terms that are leading order in $F / M^{2}$.

Due to the messenger-matter interactions these parameters are corrected. The oneand two-loop contributions at the messenger scale are summarized as

$$
\begin{aligned}
\delta \tilde{m}_{H_{U}}^{2}= & -\frac{3}{(4 \pi)^{4}}\left[\operatorname{Tr}\left(Y_{u}^{\dagger} y_{u} y_{u}^{\dagger} Y_{u}\right)+2 \operatorname{Tr}\left(Y_{u} y_{u}^{\dagger} y_{u} Y_{u}^{\dagger}\right)\right]\left|\frac{F}{M}\right|^{2} \\
\delta \tilde{m}_{H_{D}}^{2}= & -\frac{3}{(4 \pi)^{4}} \operatorname{Tr}\left(Y_{d}^{\dagger} y_{u} y_{u}^{\dagger} Y_{d}\right)\left|\frac{F}{M}\right|^{2} \\
\delta \tilde{m}_{q}^{2}= & -\frac{1}{(4 \pi)^{2}} \frac{1}{6}\left(y_{u} y_{u}^{\dagger}\right) \frac{F^{4}}{M^{6}} h(x) \\
& +\frac{1}{(4 \pi)^{4}}\left\{\left[3 \operatorname{Tr}\left(y_{u}^{\dagger} y_{u}\right)-\frac{16}{3} g_{3}^{2}-3 g_{2}^{2}-\frac{13}{15} g_{1}^{2}\right] y_{u} y_{u}^{\dagger}+3 y_{u} y_{u}^{\dagger} y_{u} y_{u}^{\dagger}+2 y_{u} Y_{u}^{\dagger} Y_{u} y_{u}^{\dagger}\right. \\
& \left.-2 Y_{u} y_{u}^{\dagger} y_{u} Y_{u}^{\dagger}+y_{u} Y_{u}^{\dagger} \operatorname{Tr}\left(3 y_{u}^{\dagger} Y_{u}\right)+Y_{u} y_{u}^{\dagger} \operatorname{Tr}\left(3 Y_{u}^{\dagger} y_{u}\right)\right\}\left|\frac{F}{M}\right|^{2}, \\
\delta \tilde{m}_{u_{R}}^{2}= & -\frac{1}{(4 \pi)^{2}} \frac{1}{3}\left(y_{u}^{\dagger} y_{u}\right) \frac{F^{4}}{M^{6}} h(x) \\
& +\frac{1}{(4 \pi)^{4}}\left\{2\left[3 \operatorname{Tr}\left(y_{u}^{\dagger} y_{u}\right)-\frac{16}{3} g_{3}^{2}-3 g_{2}^{2}-\frac{13}{15} g_{1}^{2}\right] y_{u}^{\dagger} y_{u}+6 y_{u}^{\dagger} y_{u} y_{u}^{\dagger} y_{u}+2 y_{u}^{\dagger} Y_{u} Y_{u}^{\dagger} y_{u}\right. \\
& \left.+2 y_{u}^{\dagger} Y_{d} Y_{d}^{\dagger} y_{u}-2 Y_{u}^{\dagger} y_{u} y_{u}^{\dagger} Y_{u}+2 y_{u}^{\dagger} Y_{u} \operatorname{Tr}\left(3 Y_{u}^{\dagger} y_{u}\right)+2 Y_{u}^{\dagger} y_{u} \operatorname{Tr}\left(3 y_{u}^{\dagger} Y_{u}\right)\right\}\left|\frac{F}{M}\right|^{2} \\
\delta \tilde{m}_{d_{R}}^{2}= & -\frac{1}{(4 \pi)^{4}} 2 Y_{d}^{\dagger} y_{u} y_{u}^{\dagger} Y_{d}\left|\frac{F}{M}\right|^{2}, \\
\delta m_{l}^{2}= & \delta m_{e_{R}}^{2}=0 .
\end{aligned}
$$

In the expressions for $\delta \tilde{m}_{q}^{2}$ and $\delta \tilde{m}_{u_{R}}^{2}$, the first line is the one-loop contribution suppressed by a factor of $x^{2}$ over the 2-loop and the GMSB contributions with [69]

$$
h(x)=-3 \frac{(2-x) \log (1-x)+(2+x) \log (1+x)}{x^{4}}=1+\frac{4 x^{2}}{5}+O\left(x^{4}\right) .
$$

In addition, the A-terms receive the one-loop contributions

$$
\begin{aligned}
& A_{u}^{*}=-\frac{1}{16 \pi^{2}}\left[\left(y_{u} y_{u}^{\dagger}\right) Y_{u}+2 Y_{u}\left(y_{u}^{\dagger} y_{u}\right)\right] \frac{F}{M}, \\
& A_{d}^{*}=-\frac{1}{16 \pi^{2}}\left[\left(y_{u} y_{u}^{\dagger}\right) Y_{d}\right] \frac{F}{M} .
\end{aligned}
$$




\begin{tabular}{|c|c|c|}
\hline $\mathrm{BP}$ & SOFTSUSY $m_{h}[\mathrm{GeV}]$ & FeynHiggs $m_{h}[\mathrm{GeV}]$ \\
\hline MFV-t (figure 3) & 124.6 & $123.5 \pm 2.6$ \\
\hline MFV-ct (figure 4) & 125.0 & $121.6 \pm 3.6$ \\
\hline U11 (figure 5) & 118.0 & $117.8 \pm 2.6$ \\
\hline U22L (figure 6) & 117.8 & $117.7 \pm 2.6$ \\
\hline (figure 7) & 124.6 & $120.2 \pm 5.5$ \\
\hline U32-c (figure 9) & 125.0 & $123.3 \pm 3.5$ \\
\hline U32-t (figure 10) & 124.9 & $123.3 \pm 3.0$ \\
\hline U32-ct (figure 11) & 125.7 & $120.2 \pm 2.6$ \\
\hline
\end{tabular}

Table 4. Comparison of the Higgs mass obtained from SOFTSUSY and FeynHiggs.

\section{Higgs mass validation}

Since the computation of the Higgs mass in some regions of the parameter space involves various subtleties, we also computed it with FeynHiggs [38-42] for our examples. The comparison in table 4 shows that for the selected points in parameter space, the two codes are in good agreement, with the largest discrepancies for the heavy $7 \mathrm{TeV}$ squarks spectrum of figure 7 and the mixed $\tilde{t}-\tilde{c}$ spectrum of figure 11. For spectra in the multi-TeV range, this may be due to the implementation of resummation in FeynHiggs.

\section{Calculation of flavor-violating parameters}

In this work, following [24], we have used the formula

$$
\left(\delta_{i j}^{q}\right)_{\mathrm{MM}}=\frac{\Delta \tilde{m}_{j i}^{2}}{\tilde{m}_{q}^{2}}\left(K_{M}^{q}\right)_{i j}\left(K_{M}^{q}\right)_{j j}^{*}
$$

to parameterize the flavor violation in the $M$-handed squark sector, where we ignore the LR mixing. Here $\Delta \tilde{m}_{j i}^{2}=m_{\tilde{q}_{j}}^{2}-m_{\tilde{q}_{i}}^{2}$ is the squared-mass difference of $M$-handed squarks, $\tilde{m}_{q}^{2}=\frac{1}{3} \sum_{\alpha=1}^{3} m_{\tilde{q}_{\alpha}}^{2}$ is the average mass, and $\left(K_{M}^{q}\right)_{i j}$ is the mixing appearing in the quarksquark-gluino coupling. This formula is obtained from the MIA formula [70]

$$
\sum_{a} \frac{\Delta \tilde{m}_{a}^{2}}{\tilde{m}_{q}^{2}}\left(K_{M}^{q}\right)_{i a}\left(K_{M}^{q}\right)_{j a}^{*}
$$

where $\Delta \tilde{m}_{a}^{2}=m_{\tilde{q}_{a}}^{2}-\tilde{m}_{q}^{2}$. In some of our spectra, there are noticeable differences in the values obtained for $\delta$ using the two formulae: this typically occurs when there are sizable mass splittings between or mixings of the first two generations and the third generation. Thus, as a reference, we show the values of $\left(\delta_{12}^{u}\right)_{\mathrm{MM}}$ in both of the formulae in table 5 . The other parameters $\left(\delta_{12}^{d}\right)_{\mathrm{MM}}$ and $\left(\delta_{23}^{d}\right)_{\mathrm{MM}}$ are not shown as they are far below the bounds in table 1 . All the spectra satisfy the bound on $\left|\left(\delta_{12}^{u}\right)\right|_{\mathrm{MM}}$ in table 1 , which is calculated for $\tilde{m}_{q}=1 \mathrm{TeV}$ and scales approximately linearly with $\tilde{m}_{q}$. When one takes into account this 


\begin{tabular}{|ccc|ccc|}
\hline \multicolumn{2}{|c|}{ BP } & formula & $\left|\left(\delta_{12}^{u}\right)_{L L}\right|$ & $\left|\left(\delta_{12}^{u}\right)_{R R}\right|$ & $\sqrt{\left|\left(\delta_{12}^{u}\right)_{L L}\right|\left|\left(\delta_{12}^{u}\right)_{R R}\right|}$ \\
\hline MFV-t & (figure 3) & D.1 & 0.0018 & 0.0063 & 0.0033 \\
& & D.2 & 0.0010 & $2.3 \times 10^{-5}$ & $1.6 \times 10^{-4}$ \\
\hline \multirow{2}{*}{ MFV-ct } & (figure 4) & D.1 & $3.8 \times 10^{-4}$ & 0.0077 & 0.0017 \\
& & D.2 & $6.0 \times 10^{-4}$ & $0.022 ?$ & 0.0036 \\
\hline U11 & (figure 5) & D.1 & 0.10 & 0.0011 & 0.011 \\
& & D.2 & 0.10 & 0.0011 & 0.011 \\
\hline U22L & (figure 6) & D.1 & 0.099 & 0.0020 & 0.014 \\
& & D.2 & 0.099 & 0.0020 & 0.014 \\
\hline U22 & (figure 7) & D.1 & 0.12 & 0.0023 & 0.016 \\
& & D.2 & 0.12 & 0.0023 & 0.016 \\
\hline U32-c & (figure 9) & D.1 & 0.0040 & 0.0026 & 0.0032 \\
& & D.2 & $2.7 \times 10^{-4}$ & 0.0026 & $8.4 \times 10^{-4}$ \\
\hline U32-t & (figure 10) & D.1 & $1.9 \times 10^{-4}$ & $7.4 \times 10^{-4}$ & $3.7 \times 10^{-4}$ \\
& & D.2 & $5.7 \times 10^{-5}$ & $7.4 \times 10^{-4}$ & $2.0 \times 10^{-4}$ \\
\hline U32-ct & (figure 11) & D.1 & 0.0010 & 0.0045 & 0.0022 \\
& & D.2 & $2.8 \times 10^{-4}$ & 0.0024 & $8.1 \times 10^{-4}$ \\
\hline
\end{tabular}

Table 5. $\left(\delta_{12}^{u}\right)_{\mathrm{MM}}$ for the example spectra in sections 3 and 4 given by the two formulae eq. (D.1) and eq. (D.2).

scaling, the BPs U11, U22L, and U22 have values of $\sqrt{\left|\left(\delta_{12}^{u}\right)_{L L}\right|\left|\left(\delta_{12}^{u}\right)_{R R}\right|}$ that exceed the bounds by factors of $2-3$ assuming $\mathcal{O}(1)$ phases; therefore, these examples will require mild suppression of their phases [47, 70].

Open Access. This article is distributed under the terms of the Creative Commons Attribution License (CC-BY 4.0), which permits any use, distribution and reproduction in any medium, provided the original author(s) and source are credited.

\section{References}

[1] Y. Shadmi and P.Z. Szabo, Flavored gauge-mediation, JHEP 06 (2012) 124 [arXiv: 1103.0292] [INSPIRE].

[2] M. Dine, A.E. Nelson and Y. Shirman, Low-energy dynamical supersymmetry breaking simplified, Phys. Rev. D 51 (1995) 1362 [hep-ph/9408384] [INSPIRE].

[3] M. Dine, A.E. Nelson, Y. Nir and Y. Shirman, New tools for low-energy dynamical supersymmetry breaking, Phys. Rev. D 53 (1996) 2658 [hep-ph/9507378] [INSPIRE].

[4] M. Dine, Y. Nir and Y. Shirman, Variations on minimal gauge mediated supersymmetry breaking, Phys. Rev. D 55 (1997) 1501 [hep-ph/9607397] [INSPIRE]. 
[5] Z. Chacko and E. Ponton, Yukawa deflected gauge mediation, Phys. Rev. D 66 (2002) 095004 [hep-ph/0112190] [INSPIRE].

[6] F.R. Joaquim and A. Rossi, Gauge and Yukawa mediated supersymmetry breaking in the triplet seesaw scenario, Phys. Rev. Lett. 97 (2006) 181801 [hep-ph/0604083] [INSPIRE].

[7] F.R. Joaquim and A. Rossi, Phenomenology of the triplet seesaw mechanism with Gauge and Yukawa mediation of SUSY breaking, Nucl. Phys. B 765 (2007) 71 [hep-ph/0607298] [INSPIRE].

[8] A. Brignole, F.R. Joaquim and A. Rossi, Beyond the standard seesaw: neutrino masses from Kähler operators and broken supersymmetry, JHEP 08 (2010) 133 [arXiv:1007.1942] [INSPIRE].

[9] J.L. Evans, M. Ibe and T.T. Yanagida, Relatively heavy Higgs boson in more generic gauge mediation, Phys. Lett. B 705 (2011) 342 [arXiv:1107.3006] [INSPIRE].

[10] J.L. Evans, M. Ibe and T.T. Yanagida, Probing extra matter in gauge mediation through the lightest Higgs boson mass, arXiv:1108.3437 [INSPIRE].

[11] J.L. Evans, M. Ibe, S. Shirai and T.T. Yanagida, A 125 GeV Higgs boson and muon $g-2$ in more generic gauge mediation, Phys. Rev. D 85 (2012) 095004 [arXiv:1201.2611] [INSPIRE].

[12] Z. Kang, T. Li, T. Liu, C. Tong and J.M. Yang, A heavy SM-like Higgs and a light stop from Yukawa-deflected gauge mediation, Phys. Rev. D 86 (2012) 095020 [arXiv:1203.2336] [INSPIRE].

[13] J.L. Evans, M. Ibe and T.T. Yanagida, The lightest Higgs boson mass in the MSSM with strongly interacting spectators, Phys. Rev. D 86 (2012) 015017 [arXiv:1204.6085] [INSPIRE].

[14] N. Craig, S. Knapen, D. Shih and Y. Zhao, A complete model of low-scale gauge mediation, JHEP 03 (2013) 154 [arXiv:1206.4086] [INSPIRE].

[15] A. Albaid and K.S. Babu, Higgs boson of mass $125 \mathrm{GeV}$ in GMSB models with messenger-matter mixing, Phys. Rev. D 88 (2013) 055007 [arXiv:1207.1014] [InSPIRE].

[16] M. Abdullah, I. Galon, Y. Shadmi and Y. Shirman, Flavored gauge mediation, a heavy Higgs and supersymmetric alignment, JHEP 06 (2013) 057 [arXiv: 1209.4904] [INSPIRE].

[17] P. Byakti and T.S. Ray, Burgeoning the Higgs mass to $125 \mathrm{GeV}$ through messenger-matter interactions in GMSB models, JHEP 05 (2013) 055 [arXiv:1301.7605] [INSPIRE].

[18] N. Craig, S. Knapen and D. Shih, General messenger Higgs mediation, JHEP 08 (2013) 118 [arXiv: 1302.2642] [INSPIRE].

[19] J.A. Evans and D. Shih, Surveying extended GMSB models with $m_{h}=125 \mathrm{GeV}$, JHEP 08 (2013) 093 [arXiv: 1303.0228] [INSPIRE].

[20] T. Jelinski and J. Gluza, Analytical two-loop soft mass terms of sfermions in extended GMSB models, Phys. Lett. B 751 (2015) 541 [arXiv: 1505. 07443] [INSPIRE].

[21] A. Basirnia, D. Egana-Ugrinovic, S. Knapen and D. Shih, 125 GeV Higgs from tree-level A-terms, JHEP 06 (2015) 144 [arXiv: 1501.00997] [InSPIRE].

[22] L. Calibbi, P. Paradisi and R. Ziegler, Gauge mediation beyond minimal flavor violation, JHEP 06 (2013) 052 [arXiv: 1304.1453] [INSPIRE].

[23] L. Calibbi, P. Paradisi and R. Ziegler, Lepton flavor violation in flavored gauge mediation, Eur. Phys. J. C 74 (2014) 3211 [arXiv:1408.0754] [InSPIRE]. 
[24] I. Galon, G. Perez and Y. Shadmi, Non-degenerate squarks from flavored gauge mediation, JHEP 09 (2013) 117 [arXiv: 1306.6631] [INSPIRE].

[25] R. Mahbubani, M. Papucci, G. Perez, J.T. Ruderman and A. Weiler, Light nondegenerate squarks at the LHC, Phys. Rev. Lett. 110 (2013) 151804 [arXiv:1212.3328] [INSPIRE].

[26] M. Blanke, G.F. Giudice, P. Paradisi, G. Perez and J. Zupan, Flavoured naturalness, JHEP 06 (2013) 022 [arXiv: 1302.7232] [INSPIRE].

[27] M. Blanke, B. Fuks, I. Galon and G. Perez, Gluino Meets Flavored Naturalness, JHEP 04 (2016) 044 [arXiv : 1512.03813] [INSPIRE].

[28] F. Brümmer, M. McGarrie and A. Weiler, Light third-generation squarks from flavour gauge messengers, JHEP 04 (2014) 078 [arXiv: 1312.0935] [INSPIRE].

[29] T. Jelinski and J. Pawełczyk, Masses and FCNC in flavoured GMSB scheme, arXiv:1406.4001 [INSPIRE].

[30] S. Abel and M. McGarrie, Natural supersymmetry and dynamical flavour with meta-stable vacua, JHEP 07 (2014) 145 [arXiv: 1404.1318] [INSPIRE].

[31] J.A. Evans, D. Shih and A. Thalapillil, Chiral flavor violation from extended gauge mediation, JHEP 07 (2015) 040 [arXiv: 1504.00930] [INSPIRE].

[32] M. Backović, A. Mariotti and M. Spannowsky, Signs of tops from highly mixed stops, JHEP 06 (2015) 122 [arXiv: 1504.00927] [INSPIRE].

[33] J.L. Feng, C.G. Lester, Y. Nir and Y. Shadmi, The standard model and supersymmetric flavor puzzles at the Large Hadron Collider, Phys. Rev. D 77 (2008) 076002 [arXiv:0712.0674] [INSPIRE].

[34] Y. Nir and N. Seiberg, Should squarks be degenerate?, Phys. Lett. B 309 (1993) 337 [hep-ph/9304307] [INSPIRE].

[35] D. Ghosh, P. Paradisi, G. Perez and G. Spada, CP violation tests of alignment models at LHCII, JHEP 02 (2016) 178 [arXiv:1512.03962] [INSPIRE].

[36] J.A. Evans and J. Shelton, Long-lived staus and displaced leptons at the LHC, JHEP 04 (2016) 056 [arXiv: 1601.01326] [INSPIRE].

[37] B.C. Allanach, SOFTSUSY: a program for calculating supersymmetric spectra, Comput. Phys. Commun. 143 (2002) 305 [hep-ph/0104145] [INSPIRE].

[38] S. Heinemeyer, W. Hollik and G. Weiglein, FeynHiggs: a program for the calculation of the masses of the neutral CP even Higgs bosons in the MSSM, Comput. Phys. Commun. 124 (2000) 76 [hep-ph/9812320] [INSPIRE].

[39] S. Heinemeyer, W. Hollik and G. Weiglein, The masses of the neutral CP-even Higgs bosons in the MSSM: accurate analysis at the two loop level, Eur. Phys. J. C 9 (1999) 343 [hep-ph/9812472] [INSPIRE].

[40] G. Degrassi, S. Heinemeyer, W. Hollik, P. Slavich and G. Weiglein, Towards high precision predictions for the MSSM Higgs sector, Eur. Phys. J. C 28 (2003) 133 [hep-ph/0212020] [INSPIRE].

[41] M. Frank, T. Hahn, S. Heinemeyer, W. Hollik, H. Rzehak and G. Weiglein, The Higgs boson masses and mixings of the complex MSSM in the Feynman-diagrammatic approach, JHEP 02 (2007) 047 [hep-ph/0611326] [INSPIRE]. 
[42] T. Hahn, S. Heinemeyer, W. Hollik, H. Rzehak and G. Weiglein, High-precision predictions for the light CP-even Higgs boson mass of the minimal supersymmetric standard model, Phys. Rev. Lett. 112 (2014) 141801 [arXiv:1312.4937] [INSPIRE].

[43] J. Cao, G. Eilam, K.-i. Hikasa and J.M. Yang, Experimental constraints on stop-scharm flavor mixing and implications in top-quark FCNC processes, Phys. Rev. D 74 (2006) 031701 [hep-ph/0604163] [INSPIRE].

[44] K. Kowalska, Phenomenology of SUSY with general flavour violation, JHEP 09 (2014) 139 [arXiv: 1406.0710] [INSPIRE].

[45] A. Brignole, The supersymmetric Higgs boson with flavoured A-terms, Nucl. Phys. B 898 (2015) 644 [arXiv: 1504.03273] [INSPIRE].

[46] M.D. Goodsell, K. Nickel and F. Staub, The Higgs mass in the MSSM at two-loop order beyond minimal flavour violation, Phys. Lett. B 758 (2016) 18 [arXiv:1511.01904] [INSPIRE].

[47] G. Isidori, Y. Nir and G. Perez, Flavor physics constraints for physics beyond the standard model, Ann. Rev. Nucl. Part. Sci. 60 (2010) 355 [arXiv: 1002.0900] [INSPIRE].

[48] G. Raz, The mass insertion approximation without squark degeneracy, Phys. Rev. D 66 (2002) 037701 [hep-ph/0205310] [INSPIRE].

[49] L. Calibbi, I. Galon, A. Masiero, P. Paradisi and Y. Shadmi, Charged slepton flavor post the $8 \mathrm{TeV}$ LHC: a simplified model analysis of low-energy constraints and LHC SUSY searches, JHEP 10 (2015) 043 [arXiv: 1502.07753] [INSPIRE].

[50] ATLAS collaboration, Search for supersymmetry at the high luminosity LHC with the ATLAS experiment, ATL-PHYS-PUB-2014-010 (2014).

[51] CMS Collaboration, Supersymmetry discovery potential in future LHC and HL-LHC running with the CMS detector, CMS-PAS-SUS-14-012 (2014).

[52] ATLAS collaboration, ATLAS Run 1 searches for direct pair production of third-generation squarks at the Large Hadron Collider, Eur. Phys. J. C 75 (2015) 510 [arXiv:1506.08616] [INSPIRE].

[53] CMS collaboration, Search for direct pair production of scalar top quarks in the single- and dilepton channels in proton-proton collisions at $\sqrt{s}=8 \mathrm{TeV}$, submitted to JHEP, arXiv: 1602.03169 [INSPIRE].

[54] ATLAS collaboration, Search for new phenomena in final states with large jet multiplicities and missing transverse momentum with ATLAS using $\sqrt{s}=13$ TeV proton-proton collisions, Phys. Lett. B 757 (2016) 334 [arXiv:1602.06194] [InSPIRE].

[55] CMS collaboration, Search for supersymmetry in the multijet and missing transverse momentum final state in pp collisions at 13 TeV, Phys. Lett. B 758 (2016) 152 [arXiv: 1602.06581] [INSPIRE].

[56] ATLAS collaboration, Search for photonic signatures of gauge-mediated supersymmetry in $8 \mathrm{TeV}$ pp collisions with the ATLAS detector, Phys. Rev. D 92 (2015) 072001 [arXiv: 1507.05493] [INSPIRE].

[57] CMS collaboration, Search for supersymmetry in electroweak production with photons and large missing transverse energy in pp collisions at $\sqrt{s}=8$ TeV, Phys. Lett. B 759 (2016) 479 [arXiv: 1602.08772] [INSPIRE]. 
[58] Y. Nir and G. Raz, Quark squark alignment revisited, Phys. Rev. D 66 (2002) 035007 [hep-ph/0206064] [INSPIRE].

[59] ATLAS collaboration, Searches for heavy long-lived charged particles with the ATLAS detector in proton-proton collisions at $\sqrt{s}=8 \mathrm{TeV}$, JHEP 01 (2015) 068 [arXiv:1411.6795] [INSPIRE].

[60] CMS collaboration, Search for long-lived particles decaying to photons and missing energy in proton-proton collisions at $\sqrt{s}=7 \mathrm{TeV}$, Phys. Lett. B 722 (2013) 273 [arXiv:1212.1838] [INSPIRE].

[61] ATLAS collaboration, Search for direct production of charginos, neutralinos and sleptons in final states with two leptons and missing transverse momentum in pp collisions at $\sqrt{s}=8 \mathrm{TeV}$ with the ATLAS detector, JHEP 05 (2014) 071 [arXiv: 1403.5294] [INSPIRE].

[62] CMS collaboration, Searches for electroweak production of charginos, neutralinos and sleptons decaying to leptons and $W, Z$ and Higgs bosons in pp collisions at $8 \mathrm{TeV}$, Eur. Phys. J. C 74 (2014) 3036 [arXiv: 1405.7570] [InSPIRE].

[63] ATLAS collaboration, Search for supersymmetry in events containing a same-flavour opposite-sign dilepton pair, jets and large missing transverse momentum in $\sqrt{s}=8 \mathrm{TeV} \mathrm{pp}$ collisions with the ATLAS detector, Eur. Phys. J. C 75 (2015) 318 [arXiv:1503.03290] [INSPIRE].

[64] ATLAS collaboration, Search for scalar charm quark pair production in pp collisions at $\sqrt{s}=8 \mathrm{TeV}$ with the ATLAS detector, Phys. Rev. Lett. 114 (2015) 161801 [arXiv: 1501.01325] [INSPIRE].

[65] Particle Data Group collaboration, J. Beringer et al., Review of particle physics, Phys. Rev. D 86 (2012) 010001 [INSPIRE].

[66] M. Dine and A.E. Nelson, Dynamical supersymmetry breaking at low-energies, Phys. Rev. D 48 (1993) 1277 [hep-ph/9303230] [INSPIRE].

[67] S.P. Martin, Generalized messengers of supersymmetry breaking and the sparticle mass spectrum, Phys. Rev. D 55 (1997) 3177 [hep-ph/9608224] [INSPIRE].

[68] S. Dimopoulos, G.F. Giudice and A. Pomarol, Dark matter in theories of gauge mediated supersymmetry breaking, Phys. Lett. B 389 (1996) 37 [hep-ph/9607225] [INSPIRE].

[69] S.L. Dubovsky and D.S. Gorbunov, Messenger matter mixing and lepton flavor violation, Phys. Lett. B 419 (1998) 223 [hep-ph/9706272] [INSPIRE].

[70] G. Hiller, Y. Hochberg and Y. Nir, Flavor changing processes in supersymmetric models with hybrid gauge- and gravity-mediation, JHEP 03 (2009) 115 [arXiv:0812.0511] [INSPIRE]. 